\title{
FERMI-LAT SEARCH FOR PULSAR WIND NEBULAE AROUND GAMMA-RAY PULSARS
}

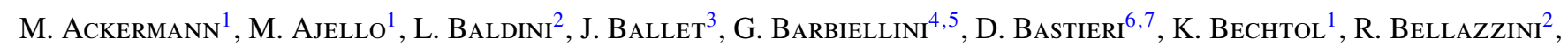
B. Berenji ${ }^{1}$, E. D. Bloom ${ }^{1}$, E. Bonamente ${ }^{8,9}$, A. W. Borgland ${ }^{1}$, A. Bouvier ${ }^{1}$, J. Bregeon ${ }^{2}$, A. Brez ${ }^{2}$, M. Brigida ${ }^{10,11}$, P. Bruel ${ }^{12}$, R. Buehler ${ }^{1}$, S. Buson ${ }^{6,7}$, G. A. Caliandro ${ }^{13}$, R. A. Cameron ${ }^{1}$, F. Camilo ${ }^{14}$, P. A. Caraveo ${ }^{15}$,


S. Ciprini ${ }^{9}$, R. Clauus ${ }^{1}$, I. Cognard ${ }^{22,23}$, J. Cohen-Tanugi ${ }^{24}$, J. Conrad ${ }^{25,26,58}$, C. D. Dermer ${ }^{19}$, A. DE Angelis ${ }^{27}$, A. De LuCa ${ }^{28}$, F. De Palma ${ }^{10,11}$, S. W. Digel ${ }^{1}$, E. do Couto e Silva ${ }^{1}$, P. S. Drell ${ }^{1}$, R. Dubois ${ }^{1}$, D. Dumora ${ }^{29}$, C. Favuzzi $^{10,11}$, W. B. Focke ${ }^{1}$, M. Frailis ${ }^{27,30}$, Y. Fukazawa ${ }^{31}$, S. FunK ${ }^{1}$, P. Fusco ${ }^{10,11}$, F. Gargano ${ }^{11}$, S. Germani ${ }^{8,9}$, N. Giglietto ${ }^{10,11}$, P. Giommi ${ }^{32}$, F. Giordano ${ }^{10,11}$, M. Girolettir ${ }^{33}$, T. GlanZman ${ }^{1}$, G. Godfrey ${ }^{1}$, I. A. Grenier ${ }^{3}$, M.-H. Grondin ${ }^{29}$, J. E. Grove ${ }^{19}$, L. Guillemot ${ }^{29,34}$, S. Guiriec ${ }^{35}$, D. Hadasch ${ }^{13}$, Y. Hanabata ${ }^{31}$, A. K. Harding ${ }^{16}$, K. HaYash ${ }^{31}$, E. Hays ${ }^{16}$, G. Hobbs $^{36}$,

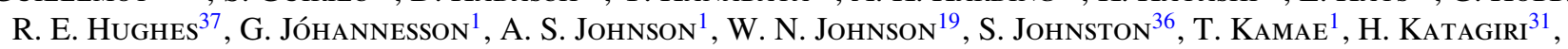
J. KATAOKa ${ }^{38}$, M. Keith ${ }^{36}$, M. KerR ${ }^{39}$, J. KNÖDlSEder ${ }^{40}$, M. Kramer ${ }^{34,41}$, M. Kuss ${ }^{2}$, J. LANDE ${ }^{1}$, L. LATronico ${ }^{2}$, S.-H. LeE $^{1}$, M. Lemoine-Goumard ${ }^{29}$, F. Longo ${ }^{4,5}$, F. Loparco ${ }^{10,11}$, M. N. Lovellette $^{19}$, P. Lubrano ${ }^{8,9}$, A. G. Lyne ${ }^{41}$, A. MaKeev ${ }^{19,20}$, M. Marelli ${ }^{15}$, M. N. Mazziotta ${ }^{11}$, J. E. McEnery ${ }^{16,42}$, J. Mehault ${ }^{24}$, P. F. Michelson ${ }^{1}$, T. Mizuno ${ }^{31}$, A. A. Moiseev ${ }^{17,42}$, C. Monte $^{10,11}$, M. E. Monzani ${ }^{1}$, A. Morsellit ${ }^{43}$, I. V. Moskalenko ${ }^{1}$, S. Murgia ${ }^{1}$, T. NAKamori ${ }^{38}$, M. Naumann-Godo ${ }^{3}$,

P. L. Nolan ${ }^{1}$, A. Noutsos ${ }^{34}$, E. Nuss ${ }^{24}$, T. Ohsugi ${ }^{44}$, A. Okumura ${ }^{45}$, J. F. Ormes ${ }^{46}$, D. Paneque ${ }^{1}$, J. H. Panetta ${ }^{1}$,

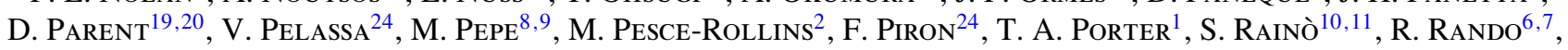

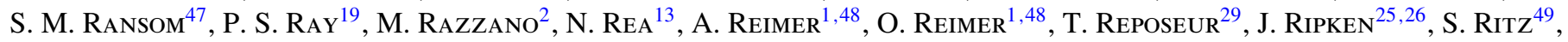

R. W. Romani ${ }^{1}$, H. F.-W. Sadrozinski ${ }^{49}$, A. Sander ${ }^{37}$, P. M. Saz Parkinson ${ }^{49}$, C. Sgrò ${ }^{2}$, E. J. Siskind ${ }^{50}$, D. A. Smith ${ }^{29}$,

P. D. Smith ${ }^{37}$, G. Spandre ${ }^{2}$, P. Spinelli ${ }^{10,11}$, M. S. Strickman ${ }^{19}$, D. J. Suson ${ }^{51}$, H. TaKahashi ${ }^{44}$, T. Takahashi ${ }^{45}$, T. TANaka ${ }^{1}$, J. B. Thayer ${ }^{1}$, J. G. Thayer ${ }^{1}$, G. Theureau ${ }^{22,23}$, D. J. Thompson ${ }^{16}$, S. E. ThorsetT ${ }^{49}$, L. Tibaldo ${ }^{3,6,7,59}$, D. F. Torres ${ }^{13,52}$,

G. Tosti ${ }^{8,9}$, A. Tramacere ${ }^{1,53,54}$, Y. Uchiyama ${ }^{1}$, T. Uehara ${ }^{31}$, T. L. Usher ${ }^{1}$, J. VAndenbroucke $^{1}$, A. Van EtTen ${ }^{1}$, V. Vasileiou ${ }^{17,18}$, N. Vilchez ${ }^{40}$, V. Vitale ${ }^{43,55}$, A. P. Waite ${ }^{1}$, P. WAng ${ }^{1}$, P. WelteVrede ${ }^{41}$, B. L. Winer ${ }^{37}$, K. S. Wood ${ }^{19}$, Z. YANG ${ }^{25,26}$, T. YLINEN ${ }^{26,56,57}$, AND M. ZIEGLER ${ }^{49}$

${ }^{1}$ W. W. Hansen Experimental Physics Laboratory, Kavli Institute for Particle Astrophysics and Cosmology, Department of Physics and SLAC National Accelerator Laboratory, Stanford University, Stanford, CA 94305, USA; joshualande@ gmail.com, ave@stanford.edu

${ }^{2}$ Istituto Nazionale di Fisica Nucleare, Sezione di Pisa, I-56127 Pisa, Italy

${ }^{3}$ Laboratoire AIM, CEA-IRFU/CNRS/Université Paris Diderot, Service d'Astrophysique, CEA Saclay, 91191 Gif sur Yvette, France

${ }^{4}$ Istituto Nazionale di Fisica Nucleare, Sezione di Trieste, I-34127 Trieste, Italy

${ }^{5}$ Dipartimento di Fisica, Università di Trieste, I-34127 Trieste, Italy

${ }^{6}$ Istituto Nazionale di Fisica Nucleare, Sezione di Padova, I-35131 Padova, Italy

7 Dipartimento di Fisica "G. Galilei," Università di Padova, I-35131 Padova, Italy

${ }^{8}$ Istituto Nazionale di Fisica Nucleare, Sezione di Perugia, I-06123 Perugia, Italy

${ }^{9}$ Dipartimento di Fisica, Università degli Studi di Perugia, I-06123 Perugia, Italy

${ }^{10}$ Dipartimento di Fisica “M. Merlin” dell'Università e del Politecnico di Bari, I-70126 Bari, Italy

${ }^{11}$ Istituto Nazionale di Fisica Nucleare, Sezione di Bari, 70126 Bari, Italy

${ }^{12}$ Laboratoire Leprince-Ringuet, École polytechnique, CNRS/IN2P3, Palaiseau, France

${ }^{13}$ Institut de Ciencies de l'Espai (IEEC-CSIC), Campus UAB, 08193 Barcelona, Spain

${ }^{14}$ Columbia Astrophysics Laboratory, Columbia University, New York, NY 10027, USA

${ }^{15}$ INAF-Istituto di Astrofisica Spaziale e Fisica Cosmica, I-20133 Milano, Italy

${ }^{16}$ NASA Goddard Space Flight Center, Greenbelt, MD 20771, USA; ahardingx@yahoo.com

${ }^{17}$ Center for Research and Exploration in Space Science and Technology (CRESST) and NASA Goddard Space Flight Center, Greenbelt, MD 20771, USA

${ }^{18}$ Department of Physics and Center for Space Sciences and Technology, University of Maryland Baltimore County, Baltimore, MD 21250, USA

${ }^{19}$ Space Science Division, Naval Research Laboratory, Washington, DC 20375, USA

${ }^{20}$ George Mason University, Fairfax, VA 22030, USA

${ }^{21}$ National Research Council Research Associate, National Academy of Sciences, Washington, DC 20001, USA

${ }^{22}$ Laboratoire de Physique et Chemie de l'Environnement, LPCE UMR 6115 CNRS, F-45071 Orléans Cedex 02, France

${ }^{23}$ Station de radioastronomie de Nançay, Observatoire de Paris, CNRS/INSU, F-18330 Nançay, France

${ }^{24}$ Laboratoire de Physique Théorique et Astroparticules, Université Montpellier 2, CNRS/IN2P3, Montpellier, France

${ }^{25}$ Department of Physics, Stockholm University, AlbaNova, SE-106 91 Stockholm, Sweden

26 The Oskar Klein Centre for Cosmoparticle Physics, AlbaNova, SE-106 91 Stockholm, Sweden

${ }^{27}$ Dipartimento di Fisica, Università di Udine and Istituto Nazionale di Fisica Nucleare, Sezione di Trieste, Gruppo Collegato di Udine, I-33100 Udine, Italy ${ }^{28}$ Istituto Universitario di Studi Superiori (IUSS), I-27100 Pavia, Italy

${ }^{29}$ Université Bordeaux 1, CNRS/IN2p3, Centre d'Études Nucléaires de Bordeaux Gradignan, 33175 Gradignan, France; grondin@ cenbg.in2p3.fr, lemoine@cenbg.in2p3.fr

${ }^{30}$ Osservatorio Astronomico di Trieste, Istituto Nazionale di Astrofisica, I-34143 Trieste, Italy

${ }^{31}$ Department of Physical Sciences, Hiroshima University, Higashi-Hiroshima, Hiroshima 739-8526, Japan

${ }^{32}$ Agenzia Spaziale Italiana (ASI) Science Data Center, I-00044 Frascati (Roma), Italy

${ }^{33}$ INAF Istituto di Radioastronomia, 40129 Bologna, Italy

${ }^{34}$ Max-Planck-Institut für Radioastronomie, Auf dem Hügel 69, 53121 Bonn, Germany

${ }^{35}$ Center for Space Plasma and Aeronomic Research (CSPAR), University of Alabama in Huntsville, Huntsville, AL 35899, USA

${ }^{36}$ Australia Telescope National Facility, CSIRO, Epping NSW 1710, Australia

${ }^{37}$ Department of Physics, Center for Cosmology and Astro-Particle Physics, The Ohio State University, Columbus, OH 43210, USA

${ }^{38}$ Research Institute for Science and Engineering, Waseda University, 3-4-1, Okubo, Shinjuku, Tokyo, 169-8555 Japan

${ }^{39}$ Department of Physics, University of Washington, Seattle, WA 98195-1560, USA

${ }^{40}$ Centre d'Étude Spatiale des Rayonnements, CNRS/UPS, BP 44346, F-30128 Toulouse Cedex 4, France 


\author{
${ }^{41}$ Jodrell Bank Centre for Astrophysics, School of Physics and Astronomy, The University of Manchester, M13 9PL, UK \\ ${ }^{42}$ Department of Physics and Department of Astronomy, University of Maryland, College Park, MD 20742, USA \\ ${ }^{43}$ Istituto Nazionale di Fisica Nucleare, Sezione di Roma "Tor Vergata," I-00133 Roma, Italy \\ ${ }^{44}$ Hiroshima Astrophysical Science Center, Hiroshima University, Higashi-Hiroshima, Hiroshima 739-8526, Japan \\ ${ }^{45}$ Institute of Space and Astronautical Science, JAXA, 3-1-1 Yoshinodai, Sagamihara, Kanagawa 229-8510, Japan \\ ${ }^{46}$ Department of Physics and Astronomy, University of Denver, Denver, CO 80208, USA \\ ${ }^{47}$ National Radio Astronomy Observatory (NRAO), Charlottesville, VA 22903, USA \\ ${ }^{48}$ Institut für Astro- und Teilchenphysik and Institut für Theoretische Physik, Leopold-Franzens-Universität Innsbruck, A-6020 Innsbruck, Austria \\ ${ }^{49}$ Santa Cruz Institute for Particle Physics, Department of Physics and Department of Astronomy and Astrophysics, University of California at Santa Cruz, \\ Santa Cruz, CA 95064, USA \\ ${ }^{50}$ NYCB Real-Time Computing Inc., Lattingtown, NY 11560-1025, USA \\ ${ }^{51}$ Department of Chemistry and Physics, Purdue University Calumet, Hammond, IN 46323-2094, USA \\ ${ }_{52}$ Institució Catalana de Recerca i Estudis Avançats (ICREA), Barcelona, Spain \\ ${ }^{53}$ Consorzio Interuniversitario per la Fisica Spaziale (CIFS), I-10133 Torino, Italy \\ ${ }^{54}$ INTEGRAL Science Data Centre, CH-1290 Versoix, Switzerland \\ 55 Dipartimento di Fisica, Università di Roma “Tor Vergata," I-00133 Roma, Italy \\ ${ }^{56}$ Department of Physics, Royal Institute of Technology (KTH), AlbaNova, SE-106 91 Stockholm, Sweden \\ ${ }^{57}$ School of Pure and Applied Natural Sciences, University of Kalmar, SE-391 82 Kalmar, Sweden \\ Received 2010 July 1; accepted 2010 October 28; published 2010 December 13
}

\begin{abstract}
The high sensitivity of the Fermi-LAT (Large Area Telescope) offers the first opportunity to study faint and extended $\mathrm{GeV}$ sources such as pulsar wind nebulae (PWNe). After one year of observation the LAT detected and identified three PWNe: the Crab Nebula, Vela-X, and the PWN inside MSH 15-52. In the meantime, the list of LAT detected pulsars increased steadily. These pulsars are characterized by high energy loss rates $(\dot{E})$ from $\sim 3 \times 10^{33} \mathrm{erg} \mathrm{s}^{-1}$ to $5 \times 10^{38} \mathrm{erg} \mathrm{s}^{-1}$ and are therefore likely to power a PWN. This paper summarizes the search for PWNe in the off-pulse windows of 54 LAT-detected pulsars using 16 months of survey observations. Ten sources show significant emission, seven of these likely being of magnetospheric origin. The detection of significant emission in the off-pulse interval offers new constraints on the $\gamma$-ray emitting regions in pulsar magnetospheres. The three other sources with significant emission are the Crab Nebula, Vela-X, and a new PWN candidate associated with the LAT pulsar PSR J1023-5746, coincident with the TeV source HESS J1023-575. We further explore the association between the HESS and the Fermi source by modeling its spectral energy distribution. Flux upper limits derived for the 44 remaining sources are used to provide new constraints on famous PWNe that have been detected at keV and/or TeV energies.
\end{abstract}

Key words: catalogs - gamma rays: general - pulsars: general

Online-only material: color figures

\section{INTRODUCTION}

Since the launch of the Fermi Gamma-Ray Space Telescope (formerly GLAST) the number of detected pulsars in the gamma-ray domain has dramatically increased. The list of Large Area Telescope (LAT) pulsars now contains 56 bright sources and certainly many more will be detected in the coming months. Yet most of the pulsar spin-down luminosity is not observed as pulsed photon emission and is instead carried away as a magnetized particle wind (Gaensler \& Slane 2006). The deceleration of the pulsar-driven wind as it sweeps up ejecta from the supernova explosion generates a termination shock at which the particles are pitch-angle scattered and further accelerated to ultra-relativistic energies. The pulsar wind nebula (PWN) emission, including synchrotron and inverse Compton components, extends across the electromagnetic spectrum from radio to $\mathrm{TeV}$ energies. PWNe studies can supply information on particle acceleration mechanisms at relativistic shocks, on the evolution of the pulsar spin down and, at later phases, on the ambient interstellar gas.

Despite the detection of 271 sources, EGRET could not firmly identify any PWNe besides the bright Crab Nebula. Most of

\footnotetext{
${ }^{58}$ Royal Swedish Academy of Sciences Research Fellow, funded by a grant from the K. A. Wallenberg Foundation.

${ }^{59}$ Partially supported by the International Doctorate on Astroparticle Physics (IDAPP) program.
}

the 170 unidentified EGRET sources at low Galactic latitudes $\left(|b| \leqslant 5^{\circ}\right)$ are associated with star-forming regions and hence may be pulsars, PWNe, supernova remnants (SNRs), winds from massive stars, or high-mass X-ray binaries (e.g., Kaaret \& Cottam 1996; Yadigaroglu \& Romani 1997; Romero et al. 1999). The early LAT observations (Abdo et al. 2010a) show that Fermi is detecting many nearby young pulsars. All FermiLAT pulsars have a high energy loss rate $(\dot{E})$, ranging from $\sim 3 \times 10^{33} \mathrm{erg} \mathrm{s}^{-1}$ to $5 \times 10^{38} \mathrm{erg} \mathrm{s}^{-1}$. About a third of these pulsars are associated with PWNe candidates observed in the $\mathrm{TeV}$ energy range by Cherenkov telescopes. These pulsars are thus likely to power a PWN detectable by Fermi. However, up to $\sim 10 \mathrm{GeV}$, the pulsed emission dominates the signal from the associated PWN, as can be seen with the example of Vela-X (Pellizzoni et al. 2009; Abdo et al. 2010c). A search for PWNe candidates around all detected Fermi-LAT pulsars thus requires that one first removes the pulsar signal, thereby selecting only the unpulsed photons.

Here, we report on the analysis of the off-pulse emission of 54 pulsars detected in the gamma-ray domain by Fermi-LAT using 16 months of survey observations: 45 pulsars ${ }^{60}$ reported in Abdo et al. (2010a), the eight new blind search pulsars (Saz Parkinson et al. 2010), and the millisecond pulsar PSR J0034-0534 (Abdo 60 The pulsar PSR J1747-2958 and its associated off-pulse emission will be
studied individually due to its proximity to the Galactic center. 
et al. 2010e). The study of the PWN in MSH 15-52, reported in Abdo et al. (2010h), did not require the selection of off-pulse photons. Therefore, its associated pulsar PSR B1509-58 is not added to our list of sources.

The primary objective of this study is to examine the properties of the off-pulse emission of each pulsar and attempt to detect the potential emission associated with its PWN. This first population study in high-energy gamma rays allows us to address astrophysical questions such as:

1. Do we see PWNe in all Fermi-LAT gamma-ray pulsars ? If not, is it because of some specific properties of the pulsar wind or of the ambient medium ?

2. What is the gamma-ray efficiency of PWNe and what physical parameters determine its value in addition to the spin-down luminosity of the pulsar?

3. What fraction of TeV PWNe candidates are detected in the Fermi-LAT energy range?

The structure of the paper is as follows. Section 2 describes the LAT, Sections 3 and 4 present the timing and spectral analyses, and the results are described in Section 5. Finally, our conclusions are summarized in Section 6.

\section{LAT DESCRIPTION AND OBSERVATIONS}

The LAT is a gamma-ray telescope that detects photons by conversion into electron-positron pairs and operates in the energy range between $20 \mathrm{MeV}$ and $300 \mathrm{GeV}$. It is made of a high-resolution converter tracker (direction measurement of the incident gamma-rays), a $\mathrm{CsI}(\mathrm{Tl})$ crystal calorimeter (energy measurement), and an anti-coincidence detector to identify the background of charged particles (Atwood et al. 2009). In comparison to EGRET, the LAT has a larger effective area $\left(\sim 8000 \mathrm{~cm}^{2}\right.$ on-axis above $\left.1 \mathrm{GeV}\right)$, a broader field of view $(\sim 2.4 \mathrm{sr})$, and superior angular resolution $(\sim 0.668 \%$ containment at $1 \mathrm{GeV}$ for events converting in the front section of the tracker). Details of the instruments and data processing are given in Atwood et al. (2009). The on-orbit calibration is described in Abdo et al. (2009a).

The following analysis used 16 months of data collected from 2008 August 4 (MJD 54682), to 2009 December 16 (MJD 55181), except for some pulsars for which portions of the observation period were rejected due to inadequate pulsar ephemerides, reported in Table 1. The Diffuse class events were selected (with the tightest background rejection). From this sample, we excluded gamma rays with a zenith angle larger than $105^{\circ}$ because of the possible contamination from Earth limb photons. We used P6_V3 post-launch instrument response functions (IRFs) that take into account pile-up and accidental coincidence effects in the detector subsystems. ${ }^{61}$

\section{TIMING ANALYSIS}

Most of the pulsars detected by Fermi-LAT are bright point sources in the gamma-ray sky up to $\sim 10 \mathrm{GeV}$, though the Vela pulsar is well detected up to $25 \mathrm{GeV}$ (Abdo et al. 2010b). The study of their associated PWNe thus requires us to assign phases to the gamma-ray photons and select only those in an offpulse window, thereby minimizing contributions from pulsars. We phase-folded photon dates using both the Fermi plug-in provided by the LAT team and distributed with the TEMPO2

61 See http://fermi.gsfc.nasa.gov/ssc/data/analysis/documentation/ Cicerone/Cicerone_LAT_IRFs/IRF_overview.html for more details. pulsar timing package, ${ }^{62}$ as well as accurate timing solutions either based on radio timing observations made at the Jodrell Bank (Hobbs et al. 2004), Nançay (Theureau et al. 2005), Parkes (Weltevrede et al. 2009) or Green Bank telescopes (Kaplan et al. 2005), or on gamma-ray data recorded by the LAT (Ray et al. 2010). Whenever possible, data from multiple radio telescopes were combined to build timing solutions, thereby improving their accuracy and expanding their time coverage.

The origins of the timing solutions used in this analysis can be found in Table 1. For each pulsar, we list the observatories that provided the data used to build the timing model. For some pulsars, we could not produce a timing solution providing accurate knowledge of the rotational phase over the whole observation range due to glitch activity. In these cases, the time intervals over which we lost phase-coherence were rejected. These intervals are given in the last column. Also listed in Table 1 are the pulsar distance (see Abdo et al. 2010a; Saz Parkinson et al. 2010; Theureau et al. 2010, for PSR J0248+6021) and the definition of the off-pulse region. These off-pulse intervals are chosen using the definition reported in previous Fermi-LAT studies (Abdo et al. 2010a, 2010e; Saz Parkinson et al. 2010) but narrowed slightly to minimize the contamination by pulsed photons. A few notes on these timing solutions:

1. The rms of the timing residuals is below $0.5 \%$ of the pulsar's rotational period in most cases, but ranges as high as 3.6\% for PSR J1846+0919 which has one of the lowest gammaray fluxes. This is adequate for the analysis performed for this paper, as timing solutions are used only for rejecting pulsed photons.

2. Glitch activity was observed for 12 pulsars over the time range considered here. These pulsars are labeled with a $g$ in Table 1. In all cases, it was possible to model the glitch parameters in such a way that all the timing data could be used except for PSRs J0205+6449, J1413-6205, and J1813-1246 where some data had to be rejected as shown in Table 1.

3. Timing solutions were built using radio timing data for all radio-emitting pulsars except PSRs J1124-5916, $\mathrm{J} 1741-2054$, J1907+0602, and J2032+4127. The first is very faint in radio and was more easily timed in gamma rays. The three others were discovered recently (Camilo et al. 2009; Abdo et al. 2010g) and radio timing observations were therefore unavailable for most of the gamma-ray data considered here. For pulsars without radio emission, timing solutions were built using the data recorded by the Fermi-LAT only.

\section{ANALYSIS OF THE Fermi-LAT DATA}

The spectral analysis was performed using a maximumlikelihood method (Mattox et al. 1996) implemented in the Fermi Science Support Center science tools as the "gtlike" code. This tool fits a source model to the data along with models for the diffuse backgrounds. Owing to uncertainties in the instrument performance still under investigation at low energies, only events in the $100 \mathrm{MeV}-100 \mathrm{GeV}$ energy band are analyzed. We used the map cube file gll_iem_v02.fit to model the Galactic diffuse emission together with the corresponding tabulated model isotropic_iem_v02.txt for the extragalactic diffuse and the residual instrument emission. ${ }^{63}$ The off-pulse spectra were

\footnotetext{
62 http://sourceforge.net/projects/tempo2/

63 Available from http://fermi.gsfc.nasa.gov/ssc/data/access/lat/ BackgroundModels.html
} 
Table 1

Observatories, Off-pulse Definitions, and Distances of the 54 Pulsars Analyzed

\begin{tabular}{|c|c|c|c|c|}
\hline PSR & ObsID & Off-pulse Definition & Distance $(\mathrm{kpc})$ & Observation Period Rejected (MJD) \\
\hline $\mathrm{J} 0007+7303^{g}$ & $\mathrm{~L}$ & $0.4-0.8$ & $1.4 \pm 0.3$ & \\
\hline J0030+0451 & $\mathrm{N}$ & $0.7-1.1$ & $0.300 \pm 0.090$ & \\
\hline J0034-0534 & $\mathrm{N}$ & $0.45-0.85$ & & \\
\hline $\mathrm{J} 0205+6449^{g}$ & G, J & $0.7-1.0$ & $2.6-3.2$ & $54870-54940$ \\
\hline $\mathrm{J} 0218+4232$ & $\mathrm{~N}$ & $0.9-1.1$ & $2.5-4$ & \\
\hline $\mathrm{J} 0248+6021^{g}$ & $\mathrm{~N}$ & $0.7-1.1$ & $2.0 \pm 0.2$ & $55161-55181$ \\
\hline $\mathrm{J} 0357+32$ & $\mathrm{~L}$ & $0.35-0.85$ & & \\
\hline J0437 - 4715 & $\mathrm{P}$ & $0.7-1.2$ & $0.1563 \pm 0.0013$ & \\
\hline $\mathrm{J} 0534+2200$ & $\mathrm{~N}, \mathrm{~J}$ & $0.5-0.85$ & $2.0 \pm 0.5$ & \\
\hline $\mathrm{J} 0613-0200$ & $\mathrm{~N}$ & $0.6-1.05$ & $0.48_{-0.11}^{+0.19}$ & \\
\hline $\mathrm{J} 0631+1036^{g}$ & $\mathrm{~N}, \mathrm{~J}$ & $0.9-1.15$ & $0.75-3.62$ & \\
\hline $\mathrm{J} 0633+0632$ & $\mathrm{~L}$ & $0.6-0.8$ & & \\
\hline J0633+1746 & $\mathrm{L}$ & $0.67-0.87$ & $0.250_{-0.062}^{+0.120}$ & \\
\hline J0659+1414 & $\mathrm{N}, \mathrm{J}$ & $0.45-1.0$ & $0.288_{-0.027}^{+0.033}$ & \\
\hline $\mathrm{J} 0742-2822^{g}$ & $\mathrm{~N}, \mathrm{~J}$ & $0.8-1.4$ & $2.07_{-1.07}^{+1.38}$ & \\
\hline $\mathrm{J} 0751+1807$ & $\mathrm{~N}$ & $0.7-1.05$ & $0.6_{-0.2}^{+0.6}$ & \\
\hline J0835 - 4510 & $\mathrm{P}$ & $0.7-1.0$ & $0.287_{-0.017}^{-0.2}$ & \\
\hline $\mathrm{J} 1023-5746^{g}$ & $\mathrm{~L}$ & $0.85-1.13$ & 2.4 & \\
\hline J1028 - 5819 & $\mathrm{P}$ & $0.8-1.05$ & $2.33 \pm 0.70$ & \\
\hline $\mathrm{J} 1044-5737$ & $\mathrm{~L}$ & $0.75-1.1$ & 1.5 & \\
\hline $\mathrm{J} 1048-5832$ & $\mathrm{P}$ & $0.7-1.05$ & $2.71 \pm 0.81$ & \\
\hline J1057 - 5226 & $P$ & $0.7-0.2$ & $0.72 \pm 0.2$ & \\
\hline $\mathrm{J} 1124-5916^{g}$ & $\mathrm{~L}$ & $0.92-0.08$ & $4.8_{-1.2}^{+0.7}$ & \\
\hline $\mathrm{J} 1413-6205^{g}$ & $\mathrm{~L}$ & $0.7-0.15$ & 1.4 & $54682-54743$ \\
\hline $\mathrm{J} 1418-6058$ & $\mathrm{~L}$ & $0.55-0.90$ & $2-5$ & \\
\hline $\mathrm{J} 1420-6048$ & $\mathrm{P}$ & $0.6-1.1$ & $5.6 \pm 1.7$ & \\
\hline J1429 - 5911 & $\mathrm{~L}$ & $0.85-0.1$ & 1.6 & \\
\hline$J 1459-60$ & $\mathrm{~L}$ & $0.34-0.69$ & & \\
\hline $\mathrm{J} 1509-5850$ & $\mathrm{P}$ & $0.6-1.0$ & $2.6 \pm 0.8$ & \\
\hline $\mathrm{J} 1614-2230$ & G & $0.92-1.14$ & $1.27 \pm 0.39$ & \\
\hline $\mathrm{J} 1709-4429^{g}$ & $\mathrm{P}$ & $0.65-1.1$ & $1.4-3.6$ & \\
\hline $\mathrm{J} 1718-3825$ & $\mathrm{~N}, \mathrm{P}$ & $0.65-1.15$ & $3.82 \pm 1.15$ & \\
\hline $\mathrm{J} 1732-31$ & $\mathrm{~L}$ & $0.54-0.89$ & & \\
\hline $\mathrm{J} 1741-2054$ & $\mathrm{~L}$ & $0.67-1.18$ & $0.38 \pm 0.11$ & \\
\hline $\mathrm{J} 1744-1134$ & $\mathrm{~N}$ & $0.15-0.35$ & $0.357_{-0.035}^{+0.043}$ & \\
\hline $\mathrm{J} 1809-2332$ & $\mathrm{~L}$ & $0.45-0.85$ & $1.7 \pm 1.0$ & \\
\hline $\mathrm{J} 1813-1246^{g}$ & $\mathrm{~L}$ & $0.72-0.84$ & & $55084-55181$ \\
\hline $\mathrm{J} 1826-1256$ & $\mathrm{~L}$ & $0.60-0.90$ & & \\
\hline $\mathrm{J} 1833-1034$ & $\mathrm{G}$ & $0.75-1.1$ & $4.7 \pm 0.4$ & \\
\hline $\mathrm{J} 1836+5925$ & $\mathrm{~L}$ & $0.16-0.28$ & $<0.8$ & \\
\hline J1846+0919 & $\mathrm{L}$ & $0.65-1.0$ & 1.2 & \\
\hline $\mathrm{J} 1907+06$ & $\mathrm{~L}$ & $0.51-0.91$ & & \\
\hline $\mathrm{J} 1952+3252$ & $\mathrm{~J}, \mathrm{~N}$ & $0.7-1.0$ & $2.0 \pm 0.5$ & \\
\hline $\mathrm{J} 1954+2836$ & $\mathrm{~L}$ & $0.85-0.2$ & 1.7 & \\
\hline $\mathrm{J} 1957+5033$ & $\mathrm{~L}$ & $0.6-0.05$ & 0.9 & \\
\hline $\mathrm{J} 1958+2846$ & $\mathrm{~L}$ & $0.55-0.90$ & & \\
\hline $\mathrm{J} 2021+3651^{g}$ & G & $0.75-1.05$ & $2.1_{-1.0}^{+2.1}$ & \\
\hline $\mathrm{J} 2021+4026$ & $\mathrm{~L}$ & $0.16-0.36$ & $1.5 \pm 0.45$ & \\
\hline $\mathrm{J} 2032+4127$ & $\mathrm{~L}$ & $0.30-0.45 \& 0.90-0.05$ & $1.6-3.6$ & \\
\hline $\mathrm{J} 2043+2740$ & $\mathrm{~N}, \mathrm{~J}$ & $0.68-0.08$ & $1.80 \pm 0.54$ & \\
\hline $\mathrm{J} 2055+2539$ & $\mathrm{~L}$ & $0.6-0.1$ & 0.4 & \\
\hline J2124 - 3358 & $\mathrm{~N}$ & $0.1-0.5$ & $0.25_{-0.08}^{+0.25}$ & \\
\hline $\mathbf{J} 2229+6114^{g}$ & G, J & $0.68-1.08$ & $0.8-6.5$ & \\
\hline $\mathrm{J} 2238+59$ & $\mathrm{~L}$ & $0.65-0.90$ & & \\
\hline
\end{tabular}

Notes. Column 1 lists the pulsars; a " $g$ " indicates that one or several glitches occurred during the observation period. For some pulsars, these glitches led us to restrict the data set to avoid any contamination of pulsed emission during the glitch: the observation period rejected in these cases is indicated in Column 5 (modified Julian day). Column 2 indicates the observatories that provided ephemerides: "G," Green Bank Telescope; “J," Lovell telescope at Jodrell Bank; "L," Large Area Telescope; "N," Nançay Radio Telescope; "P," Parkes radio telescope. Column 3 lists the off-pulse phase range used in the spectral analysis. Column 4 presents the best-known distances of 54 the pulsars analyzed in this paper. 
fit with a power-law model assuming a point source located at the position of the pulsar. Nearby sources in the field of view are extracted from Abdo et al. (2010i) and taken into account in the study. Sources within $5^{\circ}$ of the pulsar of interest and showing a significant curvature index (Abdo et al. 2010i) were left free for the analysis assuming an exponential cut-off powerlaw model, while other neighboring sources were assigned fixed power-law spectra unless the residuals showed clear indication of variability from the 1FGL catalog.

To provide better estimates of the source spectrum and search for the best PWN candidates, we split the energy range into three bands, from $100 \mathrm{MeV}$ to $1 \mathrm{GeV}, 1$ to $10 \mathrm{GeV}$, and 10 to $100 \mathrm{GeV}$. The uncertainties on the parameters were estimated using the quadratic development of the $\log$ (likelihood) around the best fit. In addition to the spectral index $\Gamma$, which is a free parameter in the fit, the important physical quantities are the photon flux $F_{0.1-100}$ (in units of photons $\mathrm{cm}^{-2} \mathrm{~s}^{-1}$ ) and the energy flux $G_{0.1-100}$ (in units of $\mathrm{erg} \mathrm{cm}^{-2} \mathrm{~s}^{-1}$ ):

$$
\begin{aligned}
F_{0.1-100} & =\int_{0.1 \mathrm{GeV}}^{100 \mathrm{GeV}} \frac{d N}{d E} d E, \\
G_{0.1-100} & =\int_{0.1 \mathrm{GeV}}^{100 \mathrm{GeV}} E \frac{d N}{d E} d E .
\end{aligned}
$$

These derived quantities are obtained from the primary fit parameters and corrected for the decreased exposure represented by the restriction to the off-pulse phase window. Their statistical uncertainties are obtained using their derivatives with respect to the primary parameters and the covariance matrix obtained from the fitting process. The estimate from the sum of the three bands is on average within $30 \%$ of the flux obtained for the global power-law fit.

An additional difficulty with this search is that we must address cases where the source flux is not significant in one or all energy bands. For each off-pulse source analyzed, gtlike provides the test statistic, $\mathrm{TS}=2 \Delta \log$ (likelihood) between models with and without the source. The TS is therefore a measure of the source significance, with TS $=25$ corresponding to a significance of just over $4.5 \sigma$. Many sources have a TS value smaller than 25 in several bands or even in the complete energy interval. In such cases, we replace the flux value from the likelihood analysis by a 95\% C.L. upper limit in Tables 2 and 3 . These upper limits were obtained using the Bayesian method proposed by Helene (1983), assuming a photon index $\Gamma=2$.

All fluxes and upper limits as well as the statistical uncertainties obtained using this procedure are summarized in Tables 2 and 3 and were all cross-checked using an analysis tool developed by the LAT team called "Sourcelike." In this method, likelihood fitting is iterated through the data set to simultaneously optimize the position and potential extension of a source, assuming spatially extended source models and taking into account nearby sources as well as Galactic diffuse and isotropic components in the fits. The results from this analysis, assuming a point-source model, are consistent with those from the likelihood analysis.

In addition to this cross-check using sourcelike, we performed a second fit to the data with gtlike incorporating the results from the first maximum likelihood analysis for all sources other than the one being considered, so it has a good representation of the surroundings of the source. This step returns a full TS map around each source of interest. These TS maps do not show any extended emission that could contaminate our source of interest (due to badly resolved diffuse background) at a TS level higher than 16.

\section{RESULTS}

PWNe candidates were selected using two different criteria:

1. $\mathrm{TS}>25$ in the whole energy range $(100 \mathrm{MeV}-100 \mathrm{GeV})$.

2 . TS $>25$ in one of the three energy bands $(100 \mathrm{MeV}-1 \mathrm{GeV}$, 1-10 GeV, 10-100 GeV).

As can be seen from Tables 2 and 3, 10 of the 54 pulsars studied here satisfy one of these detection criteria: J0034-0534, J0534+2200 associated with the Crab Nebula (Abdo et al. 2010d), J0633+1746 (Geminga), J0835-4510 associated with the Vela-X PWN (Abdo et al. 2010c), J1023-5746, $\mathrm{J} 1813-1246$ ，J1836+5925，J2021+4026，J2055+2539， and J2124-3358. A detailed study of the Crab Nebula with a model adapted to the synchrotron component at low energy was performed in Abdo et al. (2010d) and enabled its clear detection and identification by Fermi-LAT. Similarly, a detailed morphological and spectral analysis allowed the detection of the extended emission from the Vela-X PWN (Abdo et al. 2010c).

Aside from the Crab and Vela pulsars, J1023-5746 is the only candidate that shows off-pulse emission predominantly above $10 \mathrm{GeV}$, whereas the seven others are mainly detected at low energy (below $10 \mathrm{GeV}$ ) which suggests a low energy cutoff and therefore a pulsar origin. To provide further details on these seven sources and ensure that the emission detected in the off-pulse interval does not have a pulsar origin, we re-fitted all candidates using an exponential cutoff power-law spectral model; the results on the off-pulse emission of J1023-5746 are presented in Section 5.2.

\subsection{Magnetospheric Emission in the Off-pulse Window}

We explored whether the exponential cutoff power-law spectral model is preferred over a simple power-law model by computing $\mathrm{TS}_{\text {cutoff }}=2 \Delta \log$ (likelihood) (comparable to a $\chi^{2}$ distribution with 1 degree of freedom) between the models with and without the cutoff. The pulsars J0633+1746, $\mathrm{J} 1836+5925$, J2021+4026, and J2055+2539 present a significant cutoff $\left(\mathrm{TS}_{\text {cutoff }} \geqslant 9\right), \mathrm{J} 2124-3358$ being at the edge. Pulsars with $\mathrm{TS}_{\text {cutoff }}<9$ have poorly measured cutoff energies; in this case (for J1813 -1246), we report in Table 4 the fit parameters assuming a simple power law. We also determined if an extended uniform disk model (compared to the point-source hypothesis) better fits the data for each candidate. For this step, we used sourcelike and computed $\mathrm{TS}_{\text {ext }}=\mathrm{TS}_{\text {disk }}-\mathrm{TS}_{\text {point }}$. We did not find any candidates with significant extension $\left(\mathrm{TS}_{\mathrm{ext}}>9\right)$.

The Fermi-LAT spectral points for each source listed in Table 4 were obtained by dividing the $100 \mathrm{MeV}-60 \mathrm{GeV}$ range into six logarithmically spaced energy bins and performing a maximum likelihood spectral analysis in each interval, assuming a power-law shape for the source with a fixed photon index. The results, renormalized to the total phase interval, are presented in Figures 1 and 2 together with the maximum likelihood fit in the whole energy range, assuming an exponential cutoff power law (dashed blue line) or a power law (dot-dashed green line). This analysis is more reliable than a direct fit to the spectral points of Figures 1 and 2 since it accounts for Poisson statistics of the data.

Three different systematic uncertainties can affect the results derived with this analysis. The main systematic at low energy 
Table 2

Spectral Fit Results for 54 LAT-detected Pulsars

\begin{tabular}{|c|c|c|c|c|c|}
\hline PSR & TS & $\begin{array}{c}F_{0.1-100} \\
\left(10^{-9} \text { photons } \mathrm{cm}^{-2} \mathrm{~s}^{-1}\right)\end{array}$ & $\begin{array}{c}G_{0.1-100} \\
\left(10^{-12} \mathrm{erg} \mathrm{cm}^{-2} \mathrm{~s}^{-1}\right)\end{array}$ & $\Gamma$ & $\begin{array}{l}\text { Luminosity } \\
\left(10^{33} \mathrm{erg} \mathrm{s}^{-1}\right)\end{array}$ \\
\hline $\mathrm{J} 0007+7303$ & 24.3 & $<63.23$ & $<69.94$ & & $<16.40$ \\
\hline $\mathrm{J} 0030+0451$ & 3.4 & $<7.07$ & $<7.83$ & & $<0.08$ \\
\hline J0034-0534 & 29.1 & $17.26 \pm 5.70$ & $11.09 \pm 2.68$ & $2.27 \pm 0.17$ & $0.25_{-0.25}^{+0.75}$ \\
\hline J0205+6449 & 1.3 & $<11.63$ & $<12.88$ & & $<10.42-15.78$ \\
\hline J0218+4232 & 1.2 & $<12.33$ & $<13.65$ & & $<10.21-26.13$ \\
\hline J0248+6021 & 0.3 & $<7.77$ & $<8.59$ & & $<4.11$ \\
\hline $\mathrm{J} 0357+32$ & 0.0 & $<3.94$ & $<4.36$ & & $\ldots$ \\
\hline J0437-4715 & 10.7 & $<8.50$ & $<9.41$ & & $<0.03$ \\
\hline $\mathrm{J} 0534+2200^{\mathrm{a}}$ & 2775.6 & $980.00 \pm 70.00$ & $540.92 \pm 46.73$ & $2.15 \pm 0.03$ & $258.88 \pm 151.81$ \\
\hline $\mathrm{J} 0613-0200$ & 4.0 & $<6.74$ & $<7.46$ & & $<0.21$ \\
\hline J0631+1036 & 2.5 & $<18.72$ & $<20.72$ & & $<1.39-32.49$ \\
\hline J0633+0632 & 6.3 & $<32.50$ & $<35.97$ & & \\
\hline J0633+1746 & 5101.2 & $1115.54 \pm 32.31$ & $749.44 \pm 22.24$ & $2.24 \pm 0.02$ & $4.07_{-253}^{+4.42}$ \\
\hline J0659+1414 & 0.6 & $<5.04$ & $<5.58$ & & $<0.05$ \\
\hline J0742-2822 & 0.0 & $<5.99$ & $<6.63$ & & $<3.40$ \\
\hline $\mathrm{J} 0751+1807$ & 6.4 & $<9.52$ & $<10.53$ & & $<0.45$ \\
\hline $\mathrm{J} 0835-4510^{\mathrm{b}}$ & 284.3 & $405.44 \pm 26.75$ & $210.25 \pm 13.87$ & $2.30 \pm 0.10$ & $2.07_{-0.38}^{+0.41}$ \\
\hline J1023-5746 & 25.1 & $1.33 \pm 1.14$ & $27.58 \pm 13.73$ & $1.05 \pm 0.36$ & $19.01 \pm 9.46$ \\
\hline $\mathrm{J} 1028-5819$ & 20.8 & $<88.79$ & $<98.27$ & & $<63.83$ \\
\hline J1044 -5737 & 0.0 & $<11.93$ & $<13.20$ & & $<46.05$ \\
\hline $\mathrm{J} 1048-5832$ & 0.0 & $<15.33$ & $<16.96$ & & $<14.90$ \\
\hline $\mathrm{J} 1057-5226$ & 1.2 & $<10.26$ & $<11.35$ & & $<0.70$ \\
\hline J1124-5916 & 0.0 & $<12.09$ & $<13.38$ & & $<36.88$ \\
\hline $\mathrm{J} 1413-6205$ & 5.3 & $<4.83$ & $<5.34$ & & $<14.72$ \\
\hline $\mathrm{J} 1418-6058$ & 10.3 & $<77.73$ & $<86.03$ & & $<41.17-257.33$ \\
\hline $\mathrm{J} 1420-6048$ & 3.0 & $<125.98$ & $<139.42$ & & $<523.13$ \\
\hline J1429-5911 & 0.0 & $<21.26$ & $<23.53$ & & $<88.29$ \\
\hline $\mathrm{J} 1459-60$ & 1.2 & $<23.17$ & $<25.64$ & & $\ldots$ \\
\hline $\mathrm{J} 1509-5850$ & 1.1 & $<25.47$ & $<28.19$ & & $<22.80$ \\
\hline $\mathrm{J} 1614-2230$ & 5.3 & $<22.01$ & $<24.36$ & & $<9.55$ \\
\hline J1709-4429 & 16.5 & $<35.59$ & $<39.39$ & & $<61.08$ \\
\hline $\mathrm{J} 1718-3825$ & 0.0 & $<8.53$ & $<9.44$ & & $<16.48$ \\
\hline $\mathrm{J} 1732-31$ & 0.0 & $<7.40$ & $<8.19$ & & $\ldots$ \\
\hline $\mathrm{J} 1741-2054$ & 0.3 & $<10.52$ & $<11.64$ & & $<0.20$ \\
\hline J1744-1134 & 6.9 & $<27.68$ & $<30.64$ & & $<0.47$ \\
\hline $\mathrm{J} 1809-2332$ & 1.9 & $<19.19$ & $<21.25$ & & $<7.35$ \\
\hline J1813-1246 & 38.1 & $295.55 \pm 23.44$ & $119.03 \pm 9.29$ & $2.65 \pm 0.14$ & $\ldots$ \\
\hline $\mathrm{J} 1826-1256$ & 9.7 & $<145.17$ & $<160.67$ & & $\ldots$ \\
\hline $\mathrm{J} 1833-1034$ & 0.0 & $<9.38$ & $<10.38$ & & $<27.43$ \\
\hline $\mathrm{J} 1836+5925$ & 2293.6 & $579.60 \pm 28.56$ & $542.16 \pm 34.03$ & $2.07 \pm 0.03$ & $26.77 \pm 1.23$ \\
\hline $\mathrm{J} 1846+0919$ & 0.0 & $<4.79$ & $<5.30$ & & $<10.66$ \\
\hline J1907+06 & 0.7 & $<17.02$ & $<18.83$ & & $\ldots$ \\
\hline $\mathrm{J} 1952+3252$ & 2.4 & $<16.88$ & $<18.68$ & & $<8.94$ \\
\hline $\mathrm{J} 1954+2836$ & 2.4 & $<21.49$ & $<23.78$ & & $<99.04$ \\
\hline $\mathrm{J} 1957+5033$ & 0.3 & $<5.45$ & $<6.04$ & & $<7.40$ \\
\hline $\mathrm{J} 1958+2846$ & 2.6 & $<15.43$ & $<17.07$ & & $\ldots$ \\
\hline $\mathrm{J} 2021+3651$ & 15.8 & $<91.48$ & $<101.24$ & & $<53.42$ \\
\hline $\mathrm{J} 2021+4026$ & 2229.1 & $1603.0 \pm 11.2$ & $888.12 \pm 8.56$ & $2.36 \pm 0.02$ & $198.45 \pm 119.83$ \\
\hline $\mathrm{J} 2032+4127$ & 1.2 & $<154.91$ & $<171.45$ & & $<52.51-265.86$ \\
\hline $\mathrm{J} 2043+2740$ & 0.0 & $<2.71$ & $<2.99$ & & $<1.16$ \\
\hline $\mathrm{J} 2055+2539$ & 36.7 & $38.41 \pm 10.10$ & $17.59 \pm 3.34$ & $2.51 \pm 0.15$ & $2.87 \pm 1.44$ \\
\hline $\mathrm{J} 2124-3358$ & 64.6 & $22.78 \pm 6.43$ & $21.81 \pm 4.44$ & $2.06 \pm 0.14$ & $0.10_{-0.09}^{+0.22}$ \\
\hline $\mathrm{J} 2229+6114$ & 0.4 & $<14.05$ & $<15.55$ & & $<1.19-78.61$ \\
\hline $\mathrm{J} 2238+59$ & 0.0 & $<14.92$ & $<165.10$ & & $\ldots$ \\
\hline
\end{tabular}

Notes. Results of the maximum likelihood spectral fits for the off-pulse emission of LAT gamma-ray pulsars (see Section 4) between $100 \mathrm{MeV}$ and $100 \mathrm{GeV}$. PWN spectra are fitted with a power-law model (photon index $\Gamma$, photon flux $F$, and energy flux $G$ ) assuming a point source at the position of the pulsar. The test statistic (TS) for the source significance is provided in Column 2, the photon flux $F$ and the energy flux are reported in Columns 3 and 4, and the photon index is listed in Column 5 when TS $\geqslant 25$. The photon flux and energy flux obtained from the likelihood analysis are replaced by a $2 \sigma$ upper limit when TS $<25$ (assuming a photon index $\Gamma=2$ ). The total gamma-ray luminosity $L_{\gamma}$ is listed in Column 6 . The error on the photon flux and the photon index only include statistical uncertainties while the error on $L_{\gamma}$ includes the statistical uncertainties on the flux and the distance uncertainties.

${ }^{a}$ The spectral parameters of the Crab Nebula are derived using Abdo et al. (2010d).

$\mathrm{b}$ The spectral parameters are derived assuming a uniform disk morphology as described in Abdo et al. (2010c). 
Table 3

Spectral Fit Results for 54 LAT-detected Pulsars

\begin{tabular}{|c|c|c|c|c|c|c|c|c|c|}
\hline \multirow[t]{2}{*}{ PSR } & \multicolumn{3}{|c|}{$0.1-1 \mathrm{GeV}$} & \multicolumn{3}{|c|}{$1-10 \mathrm{GeV}$} & \multicolumn{3}{|c|}{$10-100 \mathrm{GeV}$} \\
\hline & TS & $\begin{array}{c}F_{0.1-1} \\
\left(10^{-9} \text { photons } \mathrm{cm}^{-2} \mathrm{~s}^{-1}\right) \\
\end{array}$ & $\Gamma$ & TS & $\begin{array}{c}F_{1-10} \\
\left(10^{-9} \text { photons } \mathrm{cm}^{-2} \mathrm{~s}^{-1}\right)\end{array}$ & $\Gamma$ & TS & $\begin{array}{c}F_{10-100} \\
\left(10^{-9} \text { photons } \mathrm{cm}^{-2} \mathrm{~s}^{-1}\right)\end{array}$ & $\Gamma$ \\
\hline $\mathrm{J} 0007+7303$ & 22.8 & $<54.39$ & & 9.2 & $<2.75$ & & 0.0 & $<0.24$ & \\
\hline $\mathrm{J} 0030+0451$ & 6.3 & $<17.11$ & & 3.7 & $<0.67$ & & 0.0 & $<0.18$ & \\
\hline J0034-0534 & 26.9 & $12.36 \pm 7.15$ & $1.47 \pm 0.60$ & 16.5 & $0.97 \pm 0.35$ & $3.14 \pm 0.78$ & 0.0 & $<0.17$ & \\
\hline J0205+6449 & 0.0 & $<12.35$ & & 2.5 & $<1.99$ & & 1.6 & $<0.30$ & \\
\hline J0218+4232 & 0.3 & $<24.46$ & & 1.1 & $<1.39$ & & 0.0 & $<0.47$ & \\
\hline J0248+6021 & 0.0 & $<5.40$ & & 0.0 & $<0.35$ & & 0.3 & $<0.25$ & \\
\hline $\mathrm{J} 0357+32$ & 0.0 & $<9.15$ & & 0.0 & $<0.56$ & & 0.0 & $<0.14$ & \\
\hline J0437-4715 & 4.1 & $<13.83$ & & 8.7 & $<0.94$ & & 0.0 & $<0.18$ & \\
\hline $\mathrm{J} 0534+2200^{\mathrm{a}}$ & 1054.5 & $785.14 \pm 45.37$ & $3.20 \pm 0.07$ & 1206.9 & $22.93 \pm 1.44$ & $1.59 \pm 0.10$ & 830.7 & $5.12 \pm 0.56$ & $1.91 \pm 0.19$ \\
\hline J0613-0200 & 5.5 & $<26.97$ & & 0.5 & $<0.72$ & & 0.0 & $<0.16$ & \\
\hline J0631+1036 & 4.6 & $<49.87$ & & 0.27 & $<0.18$ & & 0.0 & $<1.11$ & \\
\hline $\mathrm{J} 0633+0632$ & 7.4 & $<67.47$ & & 1.3 & $<2.85$ & & 0.0 & $<0.42$ & \\
\hline J0633+1746 & 3377.3 & $837.66 \pm 32.20$ & $1.81 \pm 0.05$ & 2028.4 & $65.41 \pm 3.08$ & $3.26 \pm 0.11$ & 0.0 & $<0.35$ & \\
\hline J0659+1414 & 0.5 & $<11.94$ & & 1.7 & $<0.59$ & & 0.0 & $<0.13$ & \\
\hline J0742-2822 & 0.0 & $<24.54$ & & 0.0 & $<0.94$ & & 0.0 & $<0.13$ & \\
\hline J0751+1807 & 1.7 & $<13.99$ & & 11.4 & $<1.46$ & & 0.0 & $<0.19$ & \\
\hline $\mathrm{J} 0835-4510^{\mathrm{b}}$ & 199.8 & $329.76 \pm 34.54$ & $2.15 \pm 0.11$ & 97.9 & $18.36 \pm 2.33$ & $2.22 \pm 0.20$ & 2.4 & $<0.89$ & \\
\hline $\mathrm{J} 1023-5746$ & 0.0 & $<12.55$ & & 0.9 & $<2.42$ & & 17.2 & $0.46 \pm 0.22$ & $1.02 \pm 0.73$ \\
\hline J1028-5819 & 15.8 & $<180.07$ & & 15.0 & $<7.18$ & & 0.0 & $<0.53$ & \\
\hline J1044-5737 & 0.6 & $<45.53$ & & 0.0 & $<1.46$ & & 0.0 & $<0.36$ & \\
\hline J1048-5832 & 0.0 & $<40.52$ & & 0.4 & $<2.12$ & & 0.0 & $<0.33$ & \\
\hline J1057-5226 & 0.8 & $<22.44$ & & 2.4 & $<1.33$ & & 0.0 & $<0.21$ & \\
\hline J1124-5916 & 0.0 & $<49.42$ & & 0.2 & $<2.52$ & & 0.0 & $<0.49$ & \\
\hline $\mathrm{J} 1413-6205$ & 2.8 & $<55.63$ & & 11.8 & $<6.77$ & & 0.0 & $<0.37$ & \\
\hline J1418-6058 & 5.2 & $<94.24$ & & 6.9 & $<10.28$ & & 1.1 & $<0.66$ & \\
\hline J1420-6048 & 6.7 & $<291.45$ & & 0.0 & $<9.62$ & & 1.1 & $<0.83$ & \\
\hline J1429-5911 & 0.0 & $<54.23$ & & 0.0 & $<3.25$ & & 0.0 & $<0.48$ & \\
\hline J1459-60 & 5.0 & $<68.17$ & & 0.1 & $<1.76$ & & 1.5 & $<0.56$ & \\
\hline J1509-5850 & 0.7 & $<65.47$ & & 0.4 & $<3.33$ & & 0.0 & $<0.33$ & \\
\hline J1614-2230 & 2.5 & $<34.37$ & & 7.7 & $<2.30$ & & 0.0 & $<0.43$ & \\
\hline J1709-4429 & 15.5 & $<96.67$ & & 3.3 & $<2.36$ & & 0.0 & $<0.22$ & \\
\hline J1718-3825 & 0.5 & $<56.84$ & & 0.0 & $<0.92$ & & 0.1 & $<0.24$ & \\
\hline $\mathrm{J} 1732-31$ & 0.0 & $<35.79$ & & 0.0 & $<1.09$ & & 0.0 & $<0.25$ & \\
\hline J1741-2054 & 0.9 & $<27.77$ & & 4.4 & $<1.40$ & & 0.0 & $<0.14$ & \\
\hline J1744-1134 & 10.3 & $<71.71$ & & 4.9 & $<1.92$ & & 0.0 & $<0.45$ & \\
\hline J1809-2332 & 2.8 & $<50.50$ & & 8.8 & $<2.40$ & & 1.2 & $<0.27$ & \\
\hline J1813-1246 & 32.7 & $261.21 \pm 73.15$ & $2.25 \pm 0.27$ & 16.3 & $8.43 \pm 2.57$ & $3.05 \pm 0.69$ & 3.6 & $<1.09$ & \\
\hline $\mathrm{J} 1826-1256$ & 9.5 & $<251.30$ & & 3.4 & $<5.81$ & & 0.1 & $<0.38$ & \\
\hline J1833-1034 & 0.0 & $<13.33$ & & 0.1 & $<1.67$ & & 0.2 & $<0.40$ & \\
\hline $\mathrm{J} 1836+5925$ & 1381.5 & $401.84 \pm 27.39$ & $1.56 \pm 0.09$ & 1014.1 & $51.36 \pm 3.89$ & $2.93 \pm 0.16$ & 0.0 & $<0.74$ & \\
\hline J1846+0919 & 0.0 & $<17.79$ & & 0.0 & $<0.61$ & & 0.0 & $<0.19$ & \\
\hline J1907+06 & 1.9 & $<70.95$ & & 0.9 & $<2.33$ & & 0.0 & $<0.20$ & \\
\hline $\mathrm{J} 1952+3252$ & 1.4 & $<50.81$ & & 1.3 & $<1.58$ & & 0.0 & $<0.23$ & \\
\hline $\mathrm{J} 1954+2836$ & 1.4 & $<47.98$ & & 2.6 & $<2.55$ & & 0.0 & $<0.25$ & \\
\hline $\mathrm{J} 1957+5033$ & 1.2 & $<16.35$ & & 0.2 & $<0.57$ & & 0.0 & $<0.17$ & \\
\hline $\mathrm{J} 1958+2846$ & 0.0 & $<27.14$ & & 5.3 & $<1.78$ & & 0.0 & $<0.26$ & \\
\hline $\mathrm{J} 2021+3651$ & 17.7 & $85.90 \pm 30.02$ & $1.90 \pm 0.31$ & 13.2 & $<4.50$ & & 0.0 & $<0.25$ & \\
\hline $\mathrm{J} 2021+4026$ & 1718.2 & $1344.75 \pm 55.56$ & $2.03 \pm 0.05$ & 936.2 & $73.76 \pm 3.93$ & $3.04 \pm 0.11$ & 12.16 & $<1.24$ & \\
\hline $\mathrm{J} 2032+4127$ & 3.5 & $<133.39$ & & 0.0 & $<2.08$ & & 1.3 & $<0.56$ & \\
\hline $\mathrm{J} 2043+2740$ & 0.0 & $<9.73$ & & 0.0 & $<0.76$ & & 0.0 & $<0.17$ & \\
\hline $\mathrm{J} 2055+2539$ & 35.3 & $16.06 \pm 10.90$ & $1.23 \pm 0.76$ & 23.3 & $1.53 \pm 0.42$ & $4.89 \pm 0.75$ & 0.0 & $<0.13$ & \\
\hline J2124-3358 & 16.0 & $16.75 \pm 12.17$ & $1.83 \pm 0.70$ & 56.6 & $2.41 \pm 0.54$ & $2.34 \pm 0.37$ & 0.0 & $<0.21$ & \\
\hline $\mathrm{J} 2229+6114$ & 4.2 & $<49.68$ & & 0.0 & $<1.36$ & & 0.0 & $<0.28$ & \\
\hline $\mathrm{J} 2238+59$ & 2.5 & $<55.91$ & & 0.0 & $<1.54$ & & 0.0 & $<0.38$ & \\
\hline
\end{tabular}

Notes. Results of the maximum likelihood spectral fits for the off-pulse emission of LAT gamma-ray pulsars (see Section 4). The off-pulse spectra were fit with a power-law model (photon index $\Gamma$ and photon flux $F$ ) assuming a point source at the position of the pulsar. The results for the fits in the three energy bands are reported. The test statistic (TS) for the source significance is provided in Columns $2(0.1-1 \mathrm{GeV})), 5(1-10 \mathrm{GeV})$, and $8(10-100 \mathrm{GeV})$. The photon flux $F$ for each energy band is reported in Columns 3,6, and 9; it is replaced by a $2 \sigma$ upper limit when TS $<25$ (assuming a photon index $\Gamma=2$ ). Columns 4,7 , and 10 list the photon index $\Gamma$ for each energy band when TS $\geqslant 25$. Only statistical uncertainties are reported on the photon flux and the photon index.

${ }^{a}$ The spectral parameters of the Crab Nebula are derived using Abdo et al. (2010d).

$\mathrm{b}$ The spectral parameters are derived assuming a uniform disk morphology as described in Abdo et al. (2010c). 

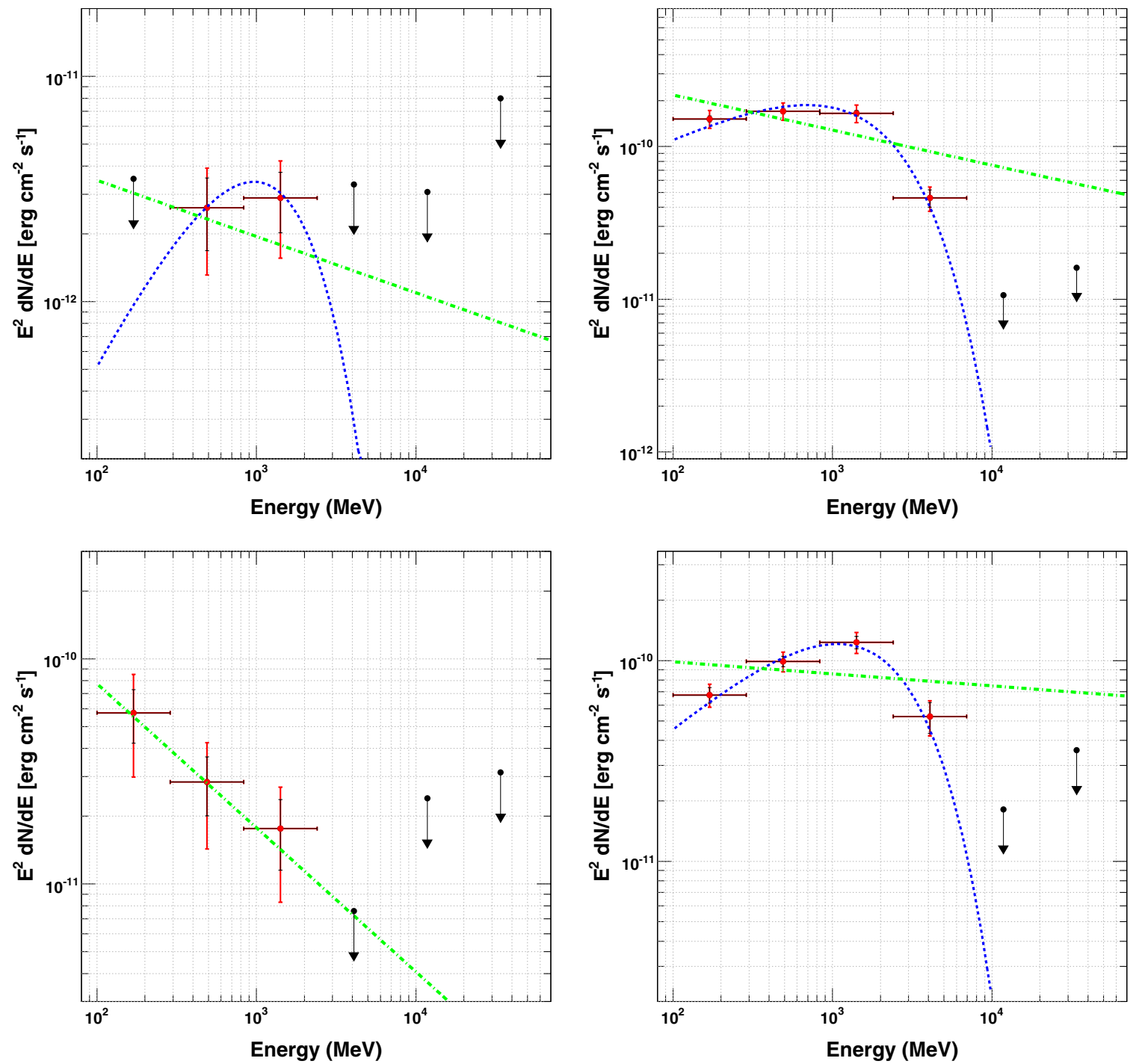

Figure 1. Spectral energy distributions of the off-pulse emission of J0034-0534 (top left), J0633+1746 (top right), J1813-1246 (bottom left), and J1836+5925 (bottom right), renormalized to the total phase interval. The LAT spectral points are obtained using the maximum likelihood method described in Section 5.1 into six logarithmically spaced energy bins. The dot-dashed green line presents the result obtained by fitting a power law to the data in the $100 \mathrm{MeV}-60 \mathrm{GeV}$ energy range using a maximum likelihood fit. The dashed blue line presents the exponential cutoff power-law model when it is favored with respect to a simple power law $\left(\mathrm{TS}_{\text {cutoff }} \geqslant 9\right.$, see Section 5.1). The statistical errors are shown in black, while the red lines take into account both the statistical and systematic errors as discussed in Section 5.1. A 95\% C.L. upper limit is computed when the statistical significance is lower than $3 \sigma$.

(A color version of this figure is available in the online journal.)

Table 4

Spectral Fitting of Pulsar Wind Nebula Candidates with Low Energy Component

\begin{tabular}{lcccc}
\hline \hline \multicolumn{1}{c}{ PSR } & $\begin{array}{c}G_{0.1-100} \\
\left(10^{-12} \mathrm{erg} \mathrm{cm}^{-2} \mathrm{~s}^{-1}\right)\end{array}$ & $\Gamma$ & \multicolumn{1}{c}{$\begin{array}{c}E_{\text {cutoff }} \\
(\mathrm{GeV})\end{array}$} & $\mathrm{TS}_{\text {cutoff }}$ \\
\hline J0034-0534 & $7.33 \pm 2.01 \pm 1.30$ & $0.62 \pm 1.05 \pm 0.27$ & $0.7 \pm 0.48 \pm 0.10$ & 9.0 \\
J0633+1746 & $544.01 \pm 13.91 \pm 54.58$ & $1.51 \pm 0.06 \pm 0.12$ & $1.41 \pm 0.14 \pm 0.09$ & 247.2 \\
$\mathrm{~J} 1813-1246$ & $116.24 \pm 22.92 \pm 79.28$ & $2.65 \pm 0.14 \pm 0.26$ & & 1.2 \\
J1836+5925 & $349.64 \pm 16.04 \pm 28.05$ & $1.33 \pm 0.10 \pm 0.06$ & $1.60 \pm 0.25 \pm 0.04$ & 99.8 \\
J2021+4026 & $737.14 \pm 21.77 \pm 125.06$ & $1.87 \pm 0.06 \pm 0.20$ & $2.24 \pm 0.37 \pm 0.51$ & 110.2 \\
J2055+2539 & $12.23 \pm 6.14 \pm 6.09$ & $0.30 \pm 1.40 \pm 0.69$ & $0.43 \pm 0.31 \pm 0.07$ & 22.4 \\
J2124-3358 & $13.27 \pm 3.02 \pm 2.77$ & $0.88 \pm 0.74 \pm 0.34$ & $1.71 \pm 1.06 \pm 0.59$ & 10.4 \\
\hline
\end{tabular}

Notes. Results of the maximum likelihood spectral fits for pulsars showing a significant signal in their off-pulse at low energy. The fits used an exponentially cutoff power-law model with the energy flux $G_{0.1-100}$, photon index $\Gamma$, and cutoff energy $E_{\text {cutoff }}$ are given in Columns $2-4$. The first errors represent the statistical error on the fit parameters, while the second ones are the systematic uncertainties as discussed in Section 5.1. The significance of an exponential cutoff (as compared to a simple power law) is indicated by $\mathrm{TS}_{\text {cutoff }}$ in Column 5 . A value $\mathrm{TS}_{\text {cutoff }}<9$ indicates that the two models are comparable and we report the fit parameters assuming a simple power-law model. 

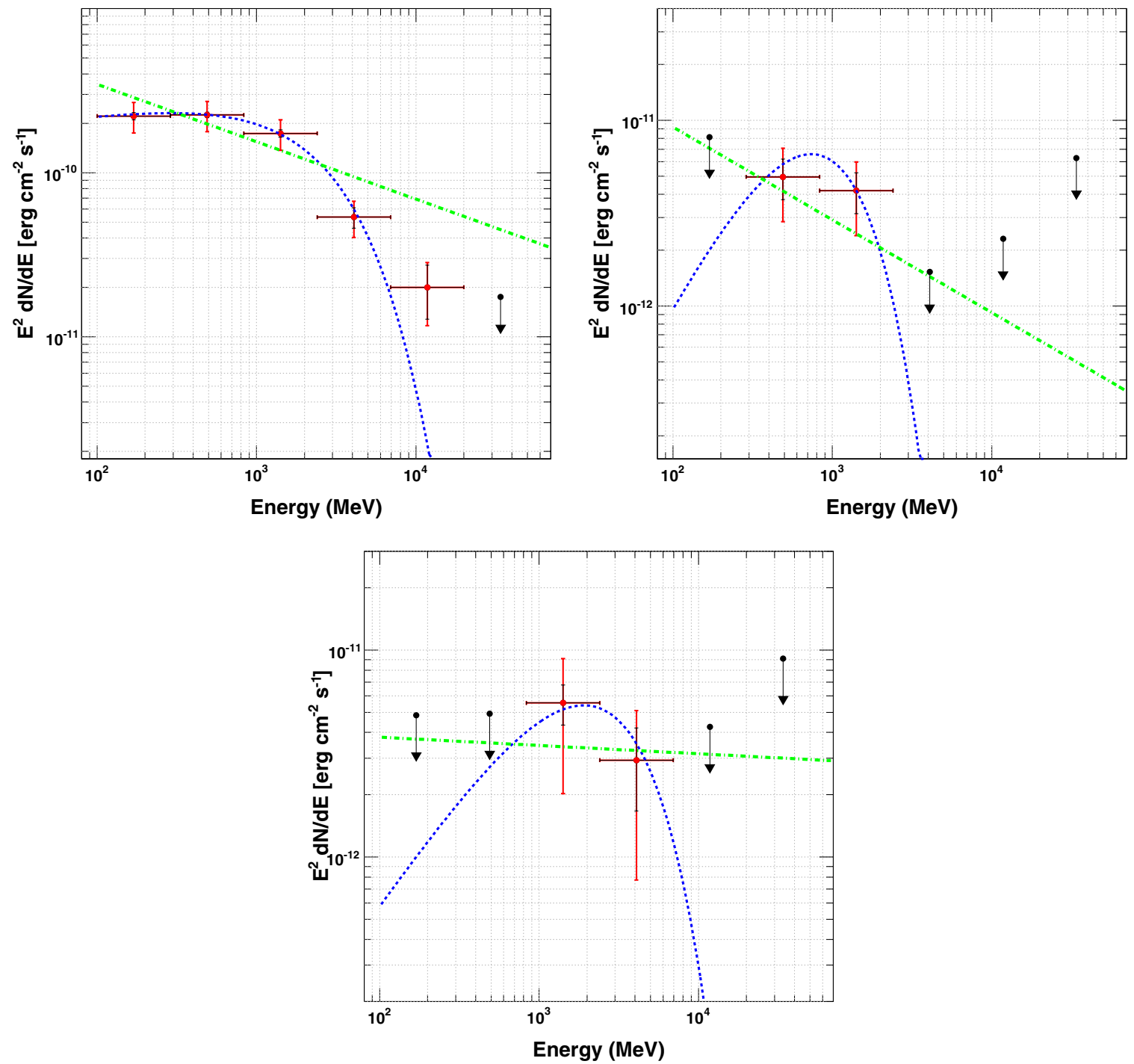

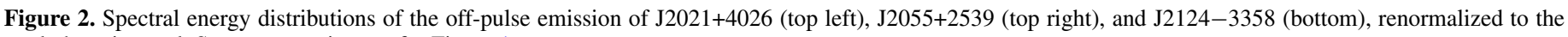
total phase interval. Same conventions as for Figure 1.

(A color version of this figure is available in the online journal.)

is due to the uncertainty in the Galactic diffuse emission. Different versions of the Galactic diffuse emission generated by GALPROP were used to estimate this error in the case of the SNRs W51C and W49 (Abdo et al. 2009b, 2010k). The difference with the best-fit diffuse model is found to be $\leqslant 6 \%$. Therefore, we estimated this systematic error by changing the normalization of the Galactic diffuse model artificially by $\pm 6 \%$. The second uncertainty, common to every source analyzed with the LAT, is due to the uncertainties in the effective area. This systematic is estimated by using modified IRFs whose effective area bracket that of our nominal IRF. These "biased" IRFs are defined by envelopes above and below the nominal dependence of the effective area with energy by linearly connecting differences of $(10 \%, 5 \%, 20 \%)$ at $\log (E)$ equal to $(2,2.75,4)$, respectively. The third systematic is related to the morphology and spectrum of the source. Taking a power-law spectral shape and a point-source morphology at the pulsar position are strong assumptions that can affect the flux and the spectral indices of the off-pulse component derived with this simple analysis, as has been demonstrated for the case of the Vela-X pulsar (Abdo et al. 2010c). A more detailed analysis of each source is beyond the scope of this paper and must be handled on a case-by-case basis. We combine the other two systematic errors in quadrature to estimate the total systematic error at each energy and propagate it through the fit model parameters reported in Table 4.

The lack of extended emission and the significant spectral cutoffs at low energies (from 0.43 to $1.71 \mathrm{GeV}$ ) suggest that the offpulse emission detected by Fermi-LAT is likely magnetospheric and that we do not observe PWNe for J0633+1736, J1836+5925, $\mathrm{J} 2021+4026$, J2055+2539, and J2124-3358. This was already suggested in previous Fermi-LAT publications on the first two pulsars, J0633+1746 (Abdo et al. 2010j) and J1836+5925 (Abdo et al. 2010f).

The cases of the Fermi-LAT pulsar PSR J1813-1246 and the millisecond pulsar J0034-0534 are harder to handle due to the limited statistics. For J0034-0534 an unpulsed component of emission from particle acceleration in the wind termination shock might be expected since this pulsar is in a binary system, though the two-pole caustic (TPC) model also predicts a faint signal in the off-pulse window of J0034-0534. In the case of J1813-1246, which shows a steep spectrum with no significant 
cutoff, we cannot rule out the PWN origin with the current statistics. We therefore cannot definitely determine the origin of the emission detected by Fermi-LAT for these two candidates.

\subsection{A Plausible Pulsar Wind Nebula Candidate Powered by PSR J1023-5746}

\subsubsection{Fermi-LAT Results on the Off-pulse Emission of PSR J1023 -5746}

In 2007, HESS reported the detection of very high energy gamma rays from an extended source, HESS J1023-575, in the direction of the young stellar cluster Westerlund 2 (Aharonian et al. 2007). Four scenarios to explain the $\mathrm{TeV}$ emission were suggested: colliding stellar winds in the WR 20a binary system (although this scenario can hardly reproduce the observed source extension of 0.18 ), collective effects of stellar winds in the Westerlund 2 cluster (although the cluster angular extent is smaller than that of the very high energy gamma ray emission), diffusive shock acceleration in the windblown bubble itself, and supersonic winds breaking out into the interstellar medium. Recently, Fermi-LAT discovered the very young (characteristic age of $4.6 \mathrm{kyr}$ ) and energetic (spin-down power of $1.1 \times 10^{37} \mathrm{erg} \mathrm{s}^{-1}$ ) pulsar J1023-5746, coincident with the TeV source HESS J1023-575 (Saz Parkinson et al. 2010).

As noted above, J1023-5746 is the only candidate that does not show any off-pulse emission below $10 \mathrm{GeV}$, whereas its signal above $10 \mathrm{GeV}$ is $>3 \sigma$. Therefore, an exponential cutoff power-law model, as used for the seven other candidates, will not represent the data properly. For these reasons, we decided to analyze this source separately.

We searched for a significant source extension using sourcelike with a uniform disk hypothesis (compared to the pointsource hypothesis). The difference in TS between the uniform disk and the point-source hypothesis is negligible which demonstrates that the two models fit equally well with the current limited statistics. We have also examined the correspondence of the gamma-ray emission with different source shapes by using gtlike with assumed multi-frequency templates. For this exercise we compared the TS values of the point source, uniform disk, and Gaussian spatial models with values derived when using a morphological template from the HESS gamma-ray excess map (Aharonian et al. 2007). We did not find any significant improvement (difference in TS $\sim 3$ ) between the different models and we therefore cannot rule out a simple point-source morphology.

To further investigate the off-pulse spectrum and avoid reliance on a given spectral shape, we derived the spectral points by dividing the $100 \mathrm{MeV}-100 \mathrm{GeV}$ range into six logarithmically spaced energy bins and performing a maximum likelihood spectral analysis in each interval assuming a point source at the position of the pulsar (as explained in Section 5.1). The result, renormalized to the total phase interval, is presented in Figure 3 with a red point and arrows. The signal is only significant above $10 \mathrm{GeV}$ and is consistent with the HESS spectral points.

\subsubsection{Broadband Modeling}

The connection between the GeV flux as observed by Fermi and the TeV flux as seen by HESS supports a common origin for the gamma-ray emission. The extension of the HESS source, the off-pulse Fermi signal, and the energetics of this young pulsar point toward a PWN origin. The very large number of PWNe detected in the TeV energy range (the most numerous

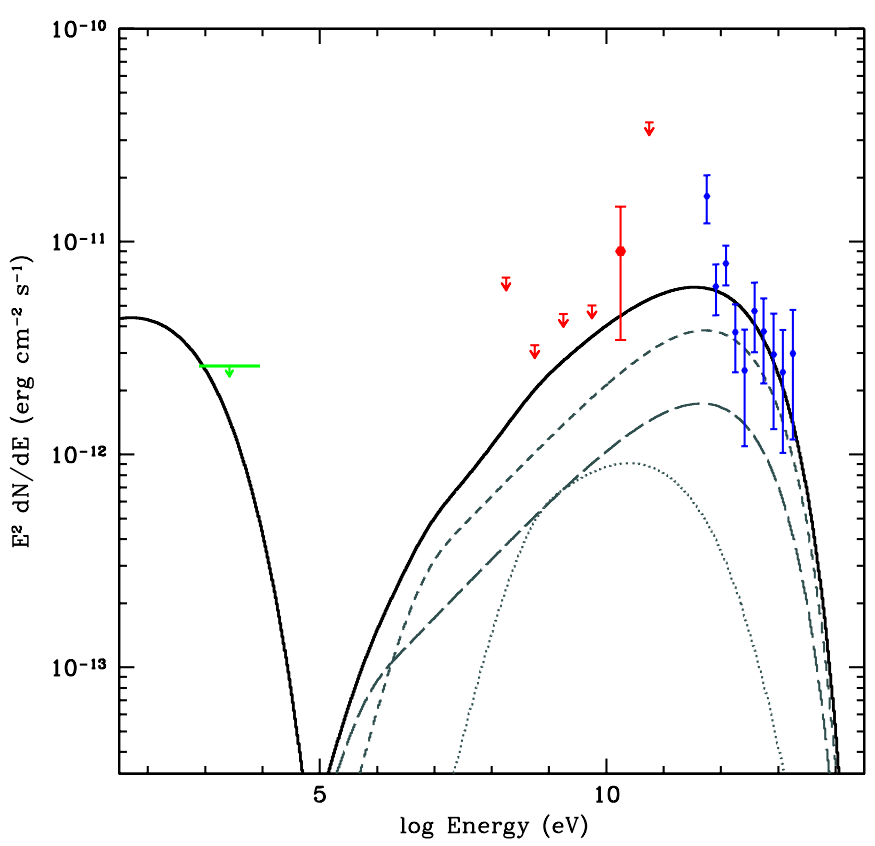

Figure 3. Spectral energy distributions of the off-pulse emission of PSR J1023-5746. The LAT spectral points (red) are obtained using the maximum likelihood method described in Section 5.2.1 in seven logarithmically spaced energy bins. A 95\% C.L. upper limit is computed when the statistical significance is lower than $3 \sigma$. The blue points represent the HESS spectral points (Aharonian et al. 2007). The Suzaku upper limit is shown with a green arrow (Fujita et al. 2009). The black line denotes the total synchrotron and Compton emission from the nebula as described in Section 5.2.2. Thin curves indicate the Compton components from scattering on the CMB (long-dashed), IR (medium-dashed), and stellar (dotted) photons.

(A color version of this figure is available in the online journal.)

class of Galactic TeV sources) and the significant number of PWNe associated with Fermi-LAT pulsars make this scenario highly probable. Analysis of $\mathrm{CO}$ emission and $21 \mathrm{~cm}$ absorption along the line of sight to Westerlund 2 gives a kinematic distance of $6.0 \pm 1.0 \mathrm{kpc}$ to the star cluster (Dame 2007). The assumption that $\mathrm{TeV}$ emission stems from the pulsar associating PSR J1023-5746 with Westerlund 2 is problematic, however. The 8 ' separation of the pulsar and the cluster imply an extremely high transverse velocity of $\sim 3000 \mathrm{~km} \mathrm{~s}^{-1}$ for a $6 \mathrm{kpc}$ distance and the pulsar's characteristic age of $4.6 \mathrm{kyr}$. In addition, the 0.18 extension of HESS J1023-757 is equivalent to 19 $\mathrm{pc}$ at a distance of $6 \mathrm{kpc}$, which predicts a very fast mean expansion velocity of $4000 \mathrm{~km} \mathrm{~s}^{-1}$ over $4.6 \mathrm{kyr}$. The pulsar pseudo-luminosity distance places it much closer at $2.4 \mathrm{kpc}$ (based on inferred beaming and gamma-ray efficiencies), though the scatter in inferred luminosities in radio-loud LAT pulsars translates to uncertainties in this estimate of the order of factors of 2-3 (Saz Parkinson et al. 2010). Both pulsar efficiency and PWN expansion velocity would be anomalously high at $6 \mathrm{kpc}$, so we adopt the pseudo-distance of $2.4 \mathrm{kpc}$. At this distance the pulsar spin-down power $\left(1.1 \times 10^{37} \mathrm{erg} \mathrm{s}^{-1}\right)$ can easily account for the Very High Energy (VHE) luminosity above $380 \mathrm{GeV}$ of $1.4 \times 10^{34} d_{2.4}^{2} \mathrm{erg} \mathrm{s}^{-1}$.

At longer wavelengths the vicinity of Westerlund 2 has undergone extensive study. Archival Chandra data indicate a faint source coincident with PSR J1023-5746, with an $\mathrm{X}$-ray index of $\Gamma=1.2 \pm 0.1$, and unabsorbed $0.5-8 \mathrm{keV}$ flux of $1.3_{-0.3}^{+0.5} \times 10^{-13} \mathrm{erg} \mathrm{cm}^{-2} \mathrm{~s}^{-1}$, though this does not affect modeling of the extended nebula. Recent Suzaku observations (Fujita et al. 2009) found no sign of diffuse non-thermal 
emission within the TeV contours and placed a $0.7-2 \mathrm{keV}$ upper limit on the diffuse flux from the entire XIS field of view of $2.6 \times 10^{-12} \mathrm{erg} \mathrm{cm}^{-2} \mathrm{~s}^{-1}$. Fujita and Collaborators also note that it is unlikely that strong X-ray emission extends beyond this field since their upper limit is consistent with the one derived using the wide HXD field $\left(34^{\prime} \times 34^{\prime}\right)$. Investigations of molecular clouds toward Westerlund 2 (Fukui et al. 2009) show features of a few $\times 10^{4} M_{\odot}$, though CO observations indicate a low density of gas (likely $n<1 \mathrm{~cm}^{-3}$ ) in the region that coincides with the bulk of the TeV emission. Radio observations of RCW 49 (the $\mathrm{H}$ II complex surrounding Westerlund 2) found a flux of $210 \mathrm{Jy}$ at $843 \mathrm{MHz}$ in the core (Whiteoak \& Uchida 1997); this provides a non-constraining upper limit on the radio flux corresponding to the gamma-ray source.

We computed SEDs (spectral energy distributions) from evolving electron populations over the lifetime of the pulsar in a series of time steps, as described in Abdo et al. (2010c). As pulsars spin down, they dissipate rotational kinetic energy via

$$
\dot{E}=I \Omega \dot{\Omega}
$$

with $\Omega$ being the angular frequency and $I$ the neutron star's moment of inertia, assumed to be $10^{45} \mathrm{~g} \mathrm{~cm}^{2}$. This energy goes into a magnetized particle wind, and for magnetic dipole spin down of the pulsar

$$
\dot{\Omega} \propto \Omega^{3} .
$$

Integrating Equation (2) yields the age of the system (Manchester \& Taylor 1977):

$$
T=\frac{P}{2 \dot{P}}\left(1-\left(\frac{P_{0}}{P}\right)^{2}\right),
$$

where $P_{0}$ is the initial spin period and $\dot{P}$ is the period derivative. For $P_{0} \ll P$ this equation reduces to the characteristic age of the pulsar $\tau_{c} \equiv P / 2 \dot{P}$. The spin-down luminosity of the pulsar evolves as (Pacini \& Salvati 1973)

$$
\dot{E}=\dot{E}_{0}\left(1+\frac{t}{\tau_{0}}\right)^{-2}
$$

with the initial spin-down timescale defined as

$$
\tau_{0} \equiv \frac{P_{0}}{2 \dot{P}_{0}}
$$

with $\dot{P}_{0}$ being the initial spin period derivative. Given that the current $P, \dot{P}$, and $\dot{E}$ are known, once an initial period is selected the age and spin-down history of the system are determined according to the equations above. We assume a particle-dominated wind such that the wind magnetization parameter $\sigma \sim 10^{-3}$. Therefore the power injected in the form of electron/positron pairs is $\dot{E}_{e}=0.999 \dot{E}$.

As the distribution of particles expand with the PWN, they lose energy through adiabatic cooling, though synchrotron cooling typically dominates for the earliest phase of PWNe evolution. We assume that the radius $R$ of the PWN scales linearly with time, and we select a magnetic field dependence of $B \propto t^{-1.5}$. Both these behaviors closely mimic the behavior of $B$ and $R$ computed by Gelfand et al. (2009) for early stage PWN evolution prior to the compression and reexpansion phases caused by the interaction of the reverse shock. Selection of appropriate photon fields is crucial to accurate determination of IC fluxes. We therefore follow Porter et al. (2006) in estimating photon fields (Cosmic Microwave Background Radiation, dust IR, and starlight) at the appropriate Galactic radii, unless local studies provide better estimates than these Galactic averages.

To compute the PWN SED we inject at each time step a power-law spectrum of relativistic electrons with a high energy exponential cutoff. We also employ a low energy cutoff of $10 \mathrm{GeV}$ for the electron spectrum, which is within the realm of minimum particle energies considered by Kennel \& Coroniti (1984). The energy content of this particle population varies with time following the pulsar spin down (Equation (4)), though we treat the index and cutoff energies as static. We then adjust the size and magnetic field according to the models described above. Finally, we calculate the subsequent particle spectrum at time $t+\delta t$ by calculating the energy loss of the particles due to adiabatic losses as well as radiation losses from synchrotron and IC (including Klein-Nishina effects). Injection (and evolution) occurs in time steps much smaller than the assumed age.

Model fitting is achieved by minimizing the $\chi^{2}$ between model and data using the downhill simplex method described in Press et al. (1992). We consider three variables: the initial spin period, electron slope, and high energy electron cutoff. With only an X-ray upper limit, the mean magnetic field within the gamma-ray source is poorly constrained, so we fix the current magnetic field to $5 \mu \mathrm{G}$ (which is the best value obtained when we allow the magnetic field to vary), or $\sim 2 \mathrm{mG}$ at pulsar birth. For each ensemble of these three variables we evolve the system over the pulsar lifetime and calculate $\chi^{2}$. The simplex routine subsequently varies the parameters of interest to minimize the fit statistic. We estimate parameter errors by computing $\chi^{2}$ for a sampling of points near the best-fit values and using these points to fit the three-dimensional ellipsoid describing the surface of $\Delta \chi^{2}=2.71$. Under the assumption of Gaussian errors, the minima and maxima of this surface give the $90 \%$ errors of the parameters.

For the assumed Galactic radius of PSR J1023-5746, dust IR photons typically peak at $\approx T=30 \mathrm{~K}$ with a density $\approx 1$ $\mathrm{eV} \mathrm{cm}^{-3}$, while stellar photons peak at $\approx T=2500 \mathrm{~K}$ with a density $\approx 2 \mathrm{eV} \mathrm{cm}^{-3}$ (Porter et al. 2006). With these photon fields (and Cosmic Microwave Background Radiation) we apply the model described above. Figure 3 indicates that IR photons dominate IC scattering above $10 \mathrm{GeV}$, with all three photon fields contributing for lower energies. For the best fit we find $\chi^{2}=13.7$ for 8 degrees of freedom, with an electron powerlaw index of $2.44 \pm 0.06$, high energy cutoff at $60 \pm 45 \mathrm{TeV}$, and initial spin period of $63 \pm 17 \mathrm{~ms}$. These parameters imply $\approx 3 \times 10^{48} \mathrm{erg}$ have been injected in the form of electrons, and an age of 3100 years.

A hadronic origin for the observed gamma rays is also possible, and we follow Kelner et al. (2006) in calculating the photons from proton-proton interactions and subsequent $\pi^{0}$ and $\eta$-meson decay. Proton-proton interactions also yield $\pi^{ \pm}$ mesons which decay into secondary electrons, which we evolve in time. The timescale for pion production via $\mathrm{p}-\mathrm{p}$ interactions is given by $\tau_{p p} \approx 1.5 \times 10^{8}\left(\mathrm{n} / 1 \mathrm{~cm}^{-3}\right)^{-1}$ years (Blumenthal 1970); this timescale is significantly greater than the expected age of the system, so the proton spectrum is treated as static. We are able to fit the gamma-ray data only if the energy in protons exceeds $2 \times 10^{50}\left(\mathrm{n} / 1 \mathrm{~cm}^{-3}\right) d_{2.4} \mathrm{erg}$, with a $\chi^{2} \approx 15$ for 8 degrees of freedom. A hadronic origin for the gammarays is therefore energetically disfavored unless the gas density is much greater than $1 \mathrm{~cm}^{-3}$ throughout the bulk of the VHE 
emitting region. Yet we cannot rule out such an origin in the confused region around Westerlund 2, even though a PWN origin is reasonable given the fit parameters discussed above.

Independent of the origin of the gamma rays, the lack of $\mathrm{X}$-rays from the immediate vicinity of PSR J1023-5746 is perplexing given its extremely high spin-down luminosity. One possibility is that electrons rapidly escape from the inner nebula into a low pressure bubble with correspondingly low magnetic field. For an electron conversion efficiency of $\sim 1$, at the current $\dot{E}$ after a mere $\approx 2$ years enough electrons are present in the inner nebula to recreate the observed X-ray flux for a $20 \mu \mathrm{G}$ field appropriate for a termination shock. This timescale is comparable to the time for particles to reach the termination shock. Post-shock flow in PWNe, as determined by torus fitting, is typically $\approx 0.7 c(\mathrm{Ng} \&$ Romani 2008$)$; at this velocity particles will traverse the $\sim 8^{\prime \prime} \mathrm{X}$-ray nebula surrounding J1023 in $\sim 0.5 d_{2.4}$ year.

\section{DISCUSSION}

\subsection{Constraints on Pulsar Modeling}

The high-quality statistics obtained with the Fermi-LAT both on the light curves and the spectra of the 54 pulsars detected allow a more detailed comparison with theoretical models than previously possible. The detection or lack of significant emission in the off-pulse interval can also be used to discriminate between the different models. Currently, there are two classes of models that differ in the location of the emission region. The first comprises polar cap (PC) models which place the emission near the magnetic poles of the neutron star (Daugherty $\&$ Harding 1996). The second class of outer magnetosphere models consists of the outer gap (OG) models (Romani 1996), in which the emission extends between the null charge surface and the light cylinder, the TPC models (Dyks \& Rudak 2003) which might be realized in slot gap (SG) acceleration models (Muslimov \& Harding 2004), in which the emission takes place between the neutron star surface and the light cylinder along the last open field lines; separatrix layer (SL) models (Bai \& Spitkovsky 2010), in which emission takes place from the neutron star surface to outside the light cylinder; and finally pairstarved polar cap (PSPC) models (Muslimov \& Harding 2004), where emission takes place throughout the entire open field region. Observations by Fermi of simple exponential cutoffs in the spectrum of Vela and other bright pulsars (Abdo et al. 2009a, 2010b), instead of super-exponential cutoffs expected in PC models, have clearly ruled out this class of model for Fermi pulsar emission. The outer magnetosphere models make different predictions for the level of off-pulse emission. Classic OG models (Romani \& Yadigaroglu 1995; Cheng et al. 2000; Romani \& Watters 2010), for which there is no emission below the null charge surface, predict no off-pulse emission except at very small inclination angles and large viewing angles near $90^{\circ}$. TPC models predict pulsed emission over most of the rotational phase at a level that depends on inclination, viewing angle, and gap width (Venter et al. 2009; Romani \& Watters 2010). In general, light curves for larger gap widths, expected for middle-aged and older pulsars in the SG model and when the viewing direction makes a large angle to the magnetic axis, have higher levels of off-pulse emission. The force-free magnetosphere SL model (Bai \& Spitkovsky 2010) also predicts light curves with off-pulse emission, since some radiation in this case also comes from below the null surface. PSPC models are expected to operate in old and millisecond pulsars and predict off-pulse emission as well (C. Venter \& A. K. Harding 2010, in preparation).

Among the 54 pulsars analyzed in this paper, only 10 show a significant signal in their off-pulse, seven of which are likely of magnetospheric origin (J0034-0534, J0633+1746, J1813-1246, J1836+5925, J2021+4026， J2055+2539, J2124-3358). Two of the seven showing off-pulse emission, J0034-0534 and J2124-3358, are millisecond pulsars. $\mathrm{J} 1836+5925$ with $\dot{E}=1.2 \times 10^{34} \mathrm{erg} \mathrm{s}^{-1}$, J2055+2539 with $\dot{E}=5 \times 10^{33} \mathrm{erg} \mathrm{s}^{-1}$, and J0633+1746 (Geminga) with $\dot{E}=$ $3.3 \times 10^{34} \mathrm{erg} \mathrm{s}^{-1}$ have among the lowest spin-down luminosities of the normal Fermi detected pulsars. While J1813-1246 and $\mathrm{J} 2021+4026$ have higher $\dot{E}\left(6.3 \times 10^{36} \mathrm{erg} \mathrm{s}^{-1}\right.$ and $1.1 \times$ $10^{35} \mathrm{erg} \mathrm{s}^{-1}$, respectively), both have unusually wide gammaray pulses.

As can be seen in the light curves presented in Figures 4 and 5, the level of off-pulse emission of these seven pulsars greatly varies. The highest levels of off-pulse emission are found for $\mathrm{J} 2021+4026$ with $\sim 40 \%, \mathrm{~J} 1836+5925$ with $\sim 35 \%$, J0034 -0534 and $\mathbf{J} 2124-3358$, with $\sim 20 \%$ of the peak heights, while lower levels are found for $\mathrm{J} 2055+2539$ and $\mathrm{J} 1813-1246$ with $\sim 10 \%$ and Geminga with $\sim 5 \%$ of the peak heights. In the case of TPC models, the highest off-pulse levels in light curves with two widely spaced peaks are produced for inclination angle $\alpha>80^{\circ}$ and viewing angle $\zeta<40^{\circ}$ or $\alpha<40^{\circ}$ and $\zeta>80^{\circ}$ (Venter et al. 2009). In the case of OG models and wide peak separations, high levels of off-pulse emission are produced only for $\alpha>85^{\circ}$ and $\zeta<30^{\circ}$ (Romani \& Watters 2010). For both types of model, $\alpha$ and $\zeta$ must be very different (i.e., we are viewing the gamma-ray emission at a large angle to the magnetic axis) and these are precisely the conditions for which our line of sight does not cross the radio beam, and for which the pulsar should be radio quiet or radio weak. In fact, all of the non-millisecond pulsars with significant levels of off-pulse emission are radio quiet (Saz Parkinson et al. 2010; Ray et al. 2010). In the case of the millisecond pulsars having large PCs and small magnetospheres, the radio beams are thought to be much larger and a significant fraction of the gamma-ray beam size. Therefore, we may still view the radio beams at large angle from the magnetic pole. The light curve of J0034-0534 shows two narrowly spaced peaks which can be fit in both TPC and OG models for $\alpha=30^{\circ}$ and $\zeta=70^{\circ}$, but off-pulse emission is predicted in this case only for TPC models ((Abdo et al. 2010e); C. Venter \& A. K. Harding 2010, in preparation). The light curve of J2124-3358 has actually been best fit with a PSPC model $\alpha=40^{\circ}$ and $\zeta=80^{\circ}$ (Venter et al. 2009), which also predicts off-pulse emission. In general, the detection of off-pulse emission in these pulsars constrains the OG solutions to a much greater degree than for TPC/SG or SL solutions.

\subsection{Constraints on Pulsar Wind Nebulae Candidates}

We searched for significant emission in the off-pulse window of 54 gamma-ray pulsars detected by Fermi-LAT and found only one convincing PWN candidate, J1023-5746 (besides the Crab Nebula and Vela-X). However, flux upper limits derived on the steady emission from the nebulae offer new constraints on sources already detected in the $\mathrm{TeV}$ range (e.g., the PWNe in the Kookaburra complex). Additionally, some PWNe were proposed by Bednarek \& Bartosik (2005) as promising sources of $\gamma$-ray emission in the $\mathrm{GeV}$ energy range, especially PSR J0205+6449 and PSR J2229+6114. We review some interesting cases in the following. 


\section{PSR J0034-0534}

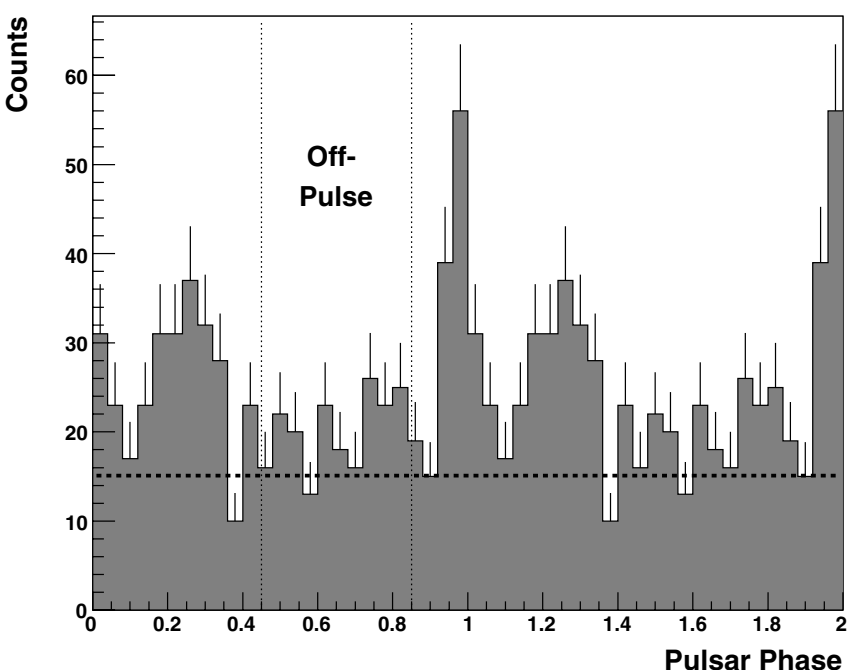

\section{PSR J1813-1246}

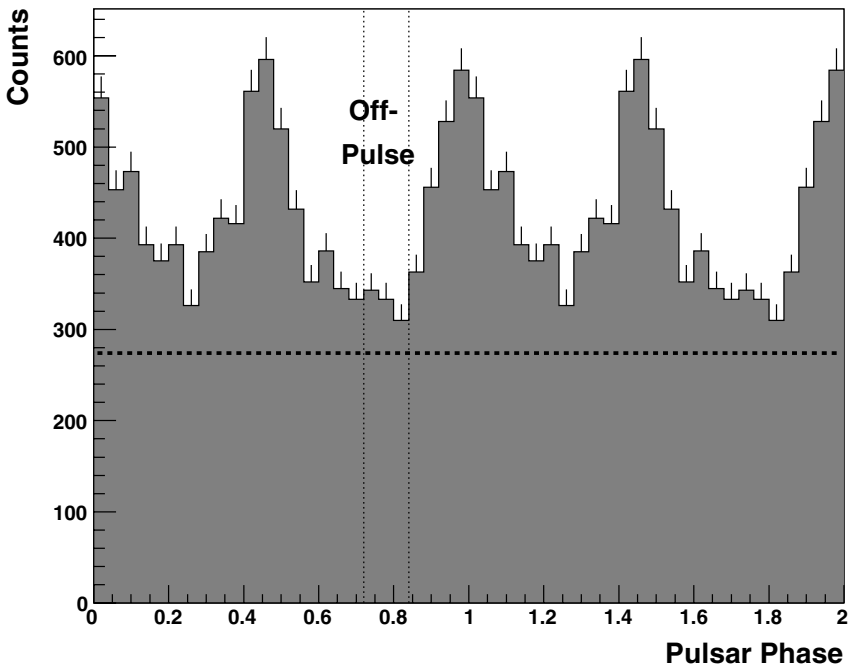

\section{PSR J0633+1746}

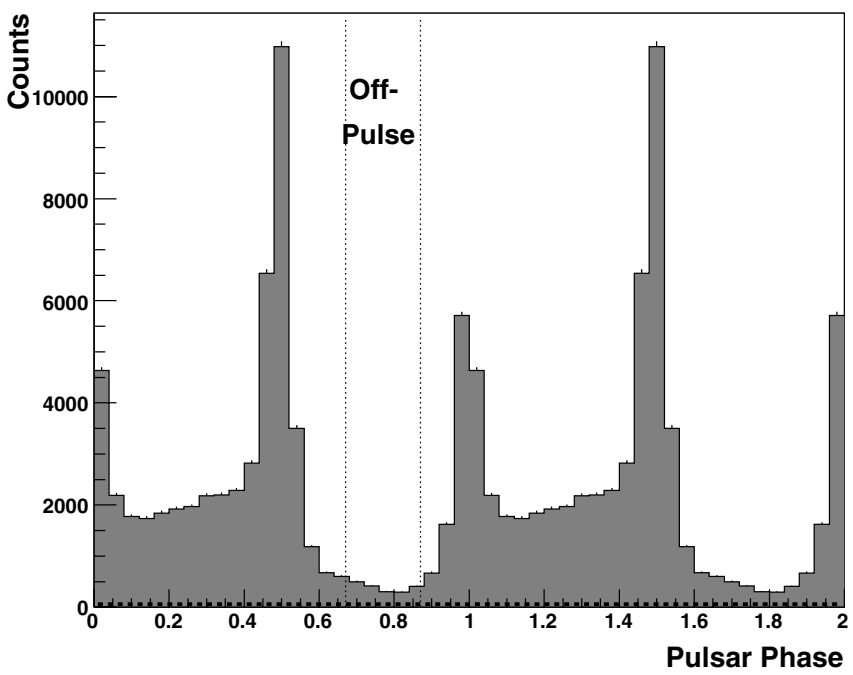

\section{PSR J1836+5925}

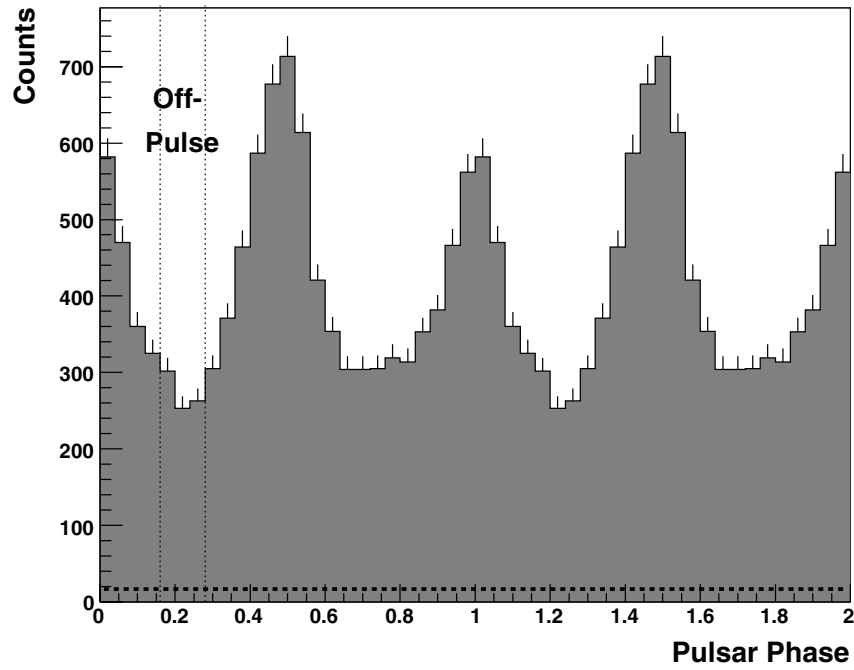

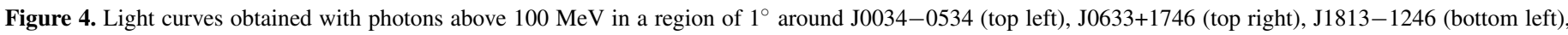

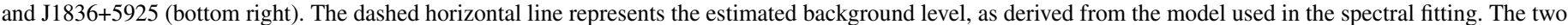
dashed vertical lines represent the definition of the off-pulse window, as defined in Table 1 . Two rotations are shown and 25 bins per rotation.

\subsubsection{PSR J0205+6449 and the PWN 3C 58}

The radio source 3C 58 was recognized early to be an SNR $(\mathrm{G} 130.7+3.1)$ and classified as a PWN by Weiler \& Panagia (1978). X-ray observations revealed a non-thermal spectrum with the photon index becoming steeper toward the outer region of the nebula (Slane et al. 2004). Flat spectrum radio emission $S_{v} \propto v^{-0.12}$ covering roughly $10^{\prime} \times 6^{\prime}$ extends up to $\sim 100 \mathrm{GHz}$ (Green 1986; Morsi \& Reich 1987; Salter et al. 1989) and corresponds well with infrared (Slane et al. 2008), and X-ray (Slane et al. 2004) morphologies. Subsequent Chandra X-ray Observatory observations detected the central pulsar of 3C 58, PSR J0205+6449. The pulsar has a very high spin-down power of $2.7 \times 10^{37} \mathrm{erg} \mathrm{s}^{-1}$ and a characteristic age of 5400 years. 3C 58 has often been associated with SN 1181 (Stephenson et al. 2002). However, recent investigations of the dynamics of the system (Chevalier 2005), and the velocities of both the radio expansion and optical knots imply an age of $\sim 2500$ years, closer to the characteristic age of PSR J0205+6449. At TeV energies, both the VERITAS and MAGIC telescopes observed this source and did not find any evidence for $\gamma$-ray emission at the position of the pulsar (Anderhub et al. 2010; Aliu 2008). The upper limits derived from their observations are consistent with the Fermi upper limits obtained in the $100 \mathrm{MeV}-100 \mathrm{GeV}$ energy range of $<12.9 \times 10^{-12} \mathrm{erg} \mathrm{cm}^{-2} \mathrm{~s}^{-1}$. This upper limit implies a non-constraining $100 \mathrm{MeV}-100 \mathrm{GeV}$ efficiency of $<4 \times 10^{-4}-6 \times 10^{-4}$ at a distance of $2.6-3.2 \mathrm{kpc}$.

\subsubsection{PSR J0633+1746 Geminga}

The Geminga pulsar is the first representative of a population of radio-quiet gamma-ray pulsars, and has been intensely studied since its discovery as a gamma-ray source by SAS-2, more than 30 years ago (Fichtel et al. 1975; Kniffen et al. 1975). The subsequent ROSAT detection of periodic X-rays from this source (Halpern \& Holt 1992) prompted a successful search for periodicity in high-energy gamma rays with EGRET (Bertsch et al. 1992) X-ray observations with XMM-Newton and Chandra observations indicate a highly structured PWN extending $\sim 50^{\prime}$ from the pulsar (Pavlov et al. 2010). 

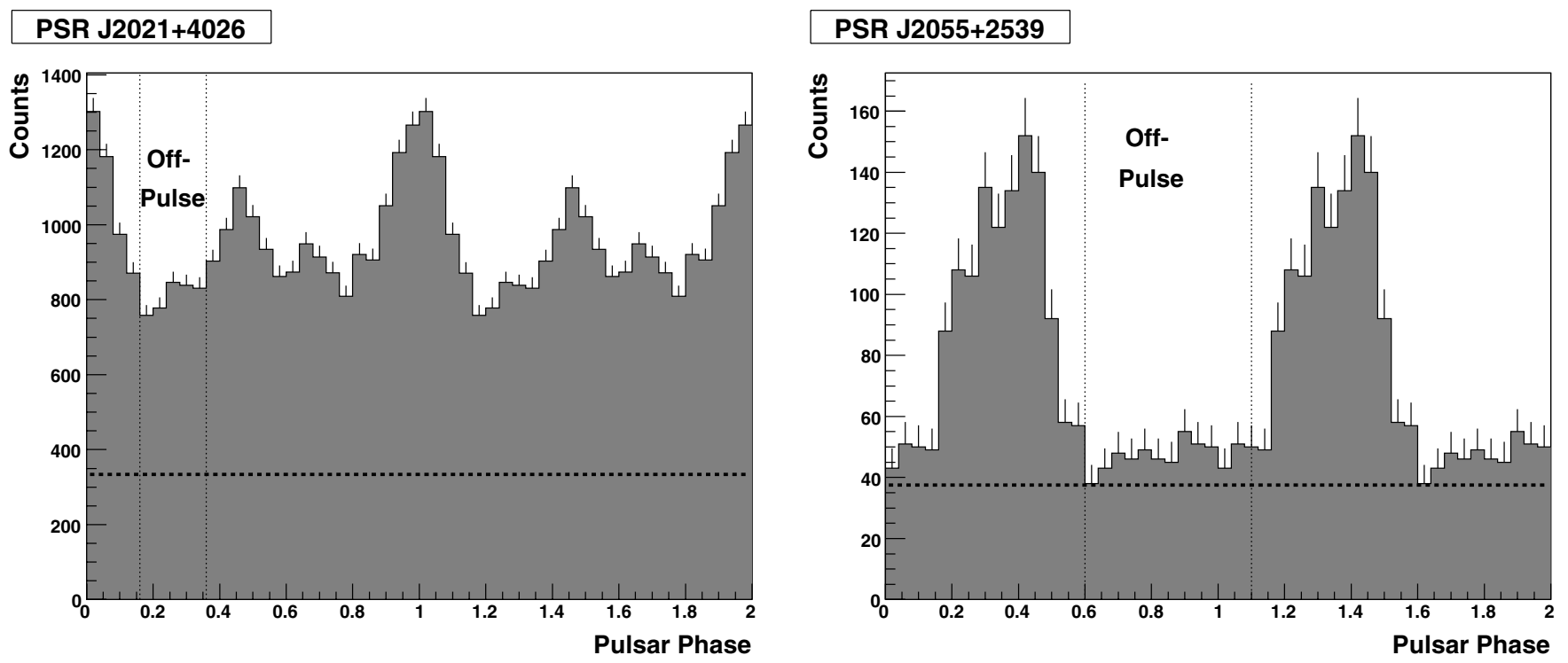

PSR J2124-3358

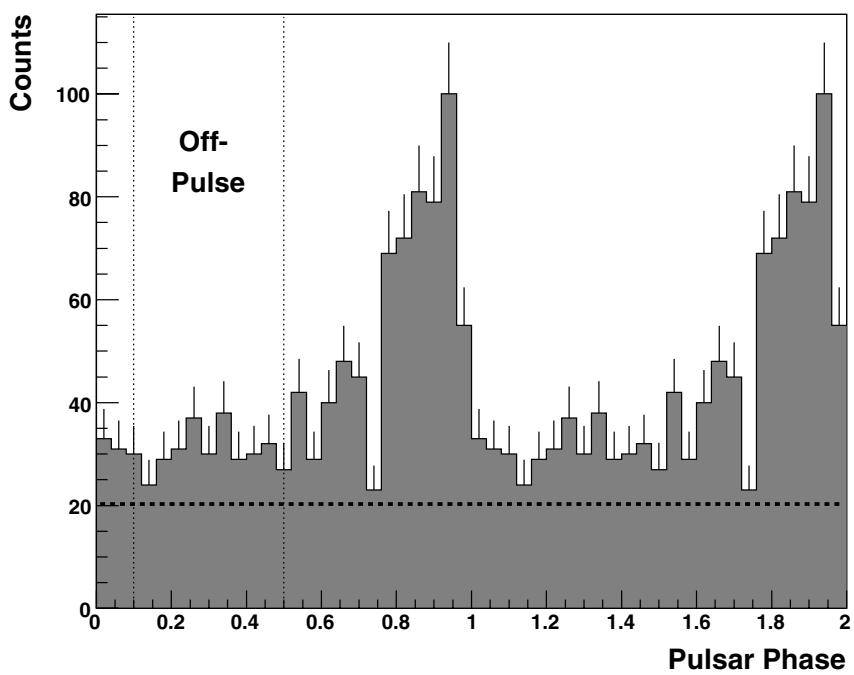

Figure 5. Light curves obtained with photons above $100 \mathrm{MeV}$ in a region of $1^{\circ}$ around J2021+4026 (top left), J2055+2539 (top right), and J2124-3358 (bottom). Same conventions as for Figure 4.

The Milagro Collaboration recently reported a $3.5 \sigma$ source coincident with Geminga of extent $\sim 2.6$, likely the result of a PWN (Abdo et al. 2009e). While VHE emission from the vicinity of this unique pulsar is certainly of interest, the Fermi upper limits on off-pulse emission above $10 \mathrm{GeV}$ are not very constraining to SED modeling of the single VHE point. The lack of data, compounded by the lack of X-ray data covering the scale of the Milagro source, renders SED modeling uninformative.

\subsubsection{PWNe in the Kookaburra Complex: the Rabbit and K3}

The complex of compact and extended radio sources called Kookaburra (Roberts et al. 1999) covers nearly $1 \mathrm{deg}^{2}$ along the Galactic plane around $l=313.4$. This region has been extensively studied to understand the nature of the unidentified EGRET source 3EG J1420-6038 (Hartman et al. 1999). In the north wing of the Kookaburra, D'Amico et al. (2001) discovered the radio pulsar PSR J1420-6048, a young energetic pulsar with period $68 \mathrm{~ms}$, characteristic age $\tau_{c}=13 \mathrm{kyr}$, and spindown power $\dot{E}=1.0 \times 10^{37} \mathrm{erg} \mathrm{s}^{-1}$. Subsequent ASCA and Chandra observations revealed an X-ray nebula surrounding the pulsar called K3. In the southwestern wing, a bright X-ray emission called the Rabbit has been proposed as a plausible PWN contributing to the $\gamma$-ray signal detected by EGRET. At $\mathrm{TeV}$ energies, the HESS Collaboration reported the detection of two bright sources coincident with the Kookaburra complex (Aharonian et al. 2006). HESS J1420-607 is centered just north of PSR J1420-6048, with best-fit position overlapping the pulsar position. HESS J1418-609 appears to correspond to the Rabbit nebula. However, the gaps in spectral coverage between HESS and EGRET did not allow a clear statement if the EGRET source is really associated with the X-ray and $\mathrm{TeV}$ emission. Finally, the $\gamma$-ray detection by Fermi-LAT of the radio-loud pulsar PSR J1420-6048 and the discovery of a radio-quiet PSR J1418-6058, likely powering the Rabbit PWN, brought a new light on this region.

In this paper, we searched for $\gamma$-ray emission in the off-pulse of both pulsars, PSR J1420-6048 and PSR J1418-6058, and did not detect any significant signal. The 95\% CL upper limits reported in Tables 2 and 3 are consistent with the prediction made by Van Etten \& Romani (2010) that do not expect any 
detection by Fermi before 10 years of observations in the most optimistic scenario for PSR J1420-6048. The VHE spectrum of the Rabbit nebula is very similar to that of K3, so similar emission mechanisms from the Rabbit would correspondingly predict little chance of Fermi detection.

\subsubsection{PSR J1833-1034 and G21.5-0.9}

G21.5-0.9 was classified as one of about 10 Crab-like SNR and predicted in 1995 to be a gamma-ray source (de Jager \& Harding 1995). Chandra observations revealed the composite nature of the remnant, consisting of a centrally peaked PWN and a $4^{\prime}$ shell (Bocchino et al. 2005; Safi-Harb et al. 2001). The $61.8 \mathrm{~ms}$ pulsar PSR J1833-1034 powering the PWN was discovered recently through its faint radio emission (Camilo et al. 2006). With a spin-down power of $\dot{E}=3.3 \times 10^{37} \mathrm{erg} \mathrm{s}^{-1}$, PSR J1833-1034 is one of the most energetic pulsars in the Galaxy. INTEGRAL observations revealed that the PWN is also bright in the hard X-ray regime (de Rosa et al. 2009). At TeV energies, G21.5-0.9 was recently detected by HESS and has a point-like nature and a hard spectrum $(\Gamma=2.08 \pm 0.22)$ (Djannati-Atai et al. 2007). The flux of this source is only $2 \%$ of that of the Crab Nebula. Although the spin-down age of this extremely energetic pulsar is $4.6 \mathrm{kyr}$, VLA measurements of the PWN expansion speed place the age at $870_{-150}^{200} \mathrm{yr}$ (Bietenholz \& Bartel 2008). Kinematic $\mathrm{H}$ I and $\mathrm{CO}$ distance measurements place G21.5-0.9 some $4.7 \mathrm{kpc}$ distant (Tian \& Leahy 2008). The lack of detection is consistent with previous modeling undertaken by de Jager et al. (2008), which predicted GeV gamma-ray flux well below the Fermi upper limits.

\subsubsection{PSR J1907+0602 and its TeV PWN}

The TeV source MGRO J1908+06 was discovered by MILAGRO at a median energy of $20 \mathrm{TeV}$ (Abdo et al. 2007) with a flux $\sim 80 \%$ of the Crab at these energies. It was subsequently detected in the $300 \mathrm{GeV}-20 \mathrm{TeV}$ range by the HESS (Aharonian et al. 2009) and VERITAS (Ward 2008) experiments. The Fermi discovery of the radio-quiet pulsar PSR J1907+0602 (Abdo et al. 2009d) within the extent of the TeV source strongly suggests that HESS J1908+063 is the PWN of PSR J1907+0602.

The upper limits derived on its off-pulse emission (see Table 2) are consistent with those previously reported in Abdo et al. (2010g) assuming a source extension of 0.3 and strongly suggest that the spectrum of HESS J1908+063 has a low-energy turnover between $20 \mathrm{GeV}$ and $300 \mathrm{GeV}$. The pulsar is offset from the HESS centroid by $15^{\prime}$, and assuming this marks the pulsar birthsite implies a velocity of $\approx 400 \mathrm{~km} \mathrm{~s}^{-1}$ for a distance of $3.2 \mathrm{kpc}$ and an age of $20 \mathrm{kyr}$. Abdo et al. (2010g) estimated an upper limit on the 2-10 keV X-ray flux from the pulsar and any arcminute-scale nebula of $10^{-13} \mathrm{erg} \mathrm{cm}^{-2} \mathrm{~s}^{-1}$, though given the HESS extension is $20^{\prime}$ we cannot constrain the magnetic field within the larger nebula. Therefore, without any data outside the VHE regime we can only conclude that a cooling break appears around $1 \mathrm{TeV}$. Determining whether that break occurs from old electrons in a low magnetic field or younger electrons in a high magnetic field requires either an X-ray detection or upper limit on the extended region.

\subsubsection{PSR J2032+4127 and TeV $2032+4130$}

TeV J2032+4130 was the first unclassified TeV source, initially detected by HEGRA (Aharonian et al. 2002), and later confirmed by MAGIC (Albert et al. 2008). Subsequent XMMNewton observations revealed a faint diffuse X-ray structure centered on the position of $\mathrm{TeV} \mathrm{J} 2032+4130$ with the same $6^{\prime}$ extension as the VHE source (Horns et al. 2007). Within the TeV error box Abdo et al. (2009d) discovered PSR J2032+4127, later confirmed by the radio detection of the pulsar (Camilo et al. 2009), hinting at a PWN origin for the TeV emission. The limited radio data and large errors on the XMM-Newton X-ray and VHE data prevent precise modeling of all PWN parameters, however, and the Fermi upper limits are approximately an order of magnitude too high to be constraining.

\subsubsection{PSR J2229+6114 and the Boomerang PWN}

The pulsar PSR J2229+6114 is as young as the Vela pulsar (characteristic age $\left.\tau_{c}=10 \mathrm{kyr}\right)$, and as energetic $(\dot{E}=2.2 \times$ $10^{37}$ erg s $^{-1}$ ), and powers the small boomerang-shaped radio and X-ray emitting PWN which is part of the SNR G106.3+2.7 (Halpern et al. 2001a). The pulsar distance estimated from X-ray absorption is $\sim 3 \mathrm{kpc}$ (Halpern et al. 2001a), while the dispersion measure used in conjunction with the NE2001 model (Cordes \& Lazio 2002) yields a distance of $7.5 \mathrm{kpc}$ (Abdo et al. 2009c); polarization studies and velocity maps of $\mathrm{H}$ I and $\mathrm{CO}$ emission imply a distance of 800 pc (Kothes et al. 2006). In this paper, we have used the range $0.8-6.5 \mathrm{kpc}$ as reported in Table 1.

At $\mathrm{TeV}$ energies, the MAGIC Collaboration placed a constraining point-source upper limit of $3.95 \times 10^{-12} \mathrm{~cm}^{-2} \mathrm{~s}^{-1}$ at the position of the pulsar (Anderhub et al. 2010). However, recently, both the MILAGRO and the VERITAS Collaboration reported a significant detection in this region. The centroid of the extended emission (full angular extent of 0.6 by 0.4 ) detected by VERITAS is located 0.4 away from the pulsar PSR J2229+6114 (Acciari et al. 2009). The signal detected by MILAGRO is spread over a broad $\sim 1^{\circ}$ area encompassing the pulsar position and the main bulk of the remnant, which does not allow a definitive association with a particular region of the SNR/pulsar complex (Abdo et al. 2009e).

Using 16 months of Fermi-LAT data, we do not report any significant detection at the position of the pulsar using its offpulse photons. The upper limits derived are compatible with the non-detection reported by the MAGIC Collaboration at $\mathrm{TeV}$ energies (Anderhub et al. 2010). An offset between the pulsar J2229+6114 and its PWN is thus required if the emission detected at $\mathrm{TeV}$ energies by VERITAS and MILAGRO is produced by the PWN. This is the case for several PWNe already detected by Cherenkov telescopes, such as HESS J1825-137 and Vela-X, and can be explained by the supernova explosion that occurred in an inhomogeneous medium leading to an asymmetric reverse shock that displaced the PWN (Blondin 2001) toward lower densities. However, the $\mathrm{TeV}$ emission detected by VERITAS is coincident with the location of molecular clouds which disfavors such a scenario and supports a hadronic origin where $\gamma$-rays are produced via proton-proton interactions within the molecular clouds.

\subsection{Population Study of Pulsar Wind Nebulae as Observed by Fermi-LAT}

Among the large sample of pulsars analyzed in this paper, two sources are firmly identified as PWNe (the Crab Nebula and Vela-X), one source is suggested as a highly plausible candidate (the emission in the off-pulse of PSR J1023-5746), in addition to the PWN in MSH 15-52 (Abdo et al. 2010h). As can be seen in Figure 6, the pulsars powering these PWNe are all young (in the range $1-10 \mathrm{kyr}$ ) and bright $\left(\dot{E} \geqslant 7 \times 10^{36} \mathrm{erg} \mathrm{s}^{-1}\right)$; their associated PWNe are detected by Cherenkov telescopes in the $\mathrm{TeV}$ range. Remarkably, these four PWNe candidates 


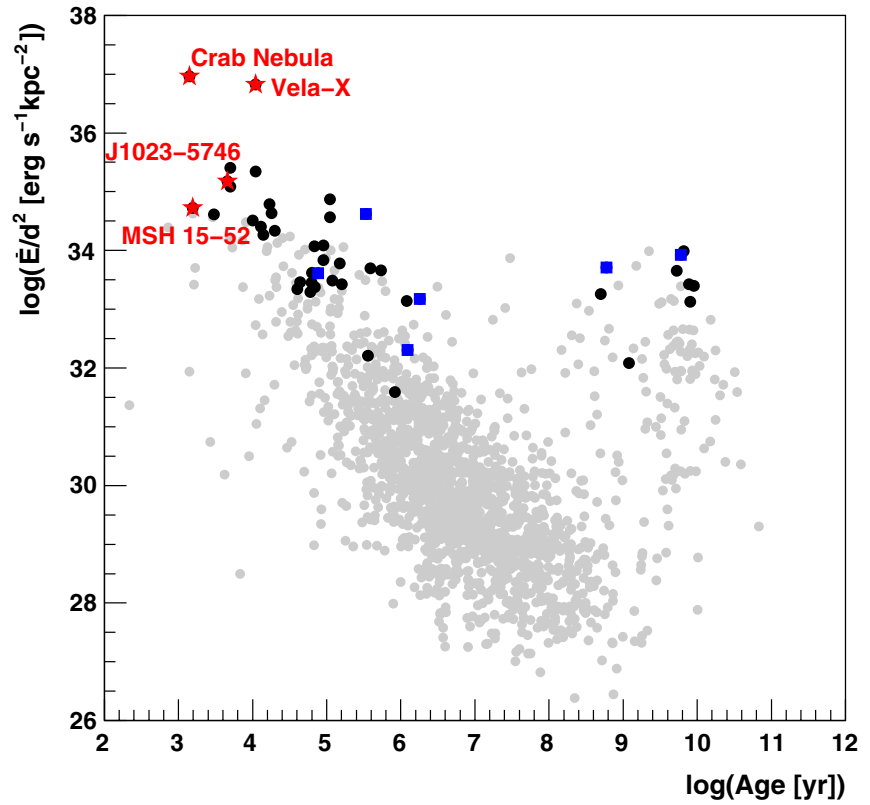

Figure 6. Spin-down flux at Earth as a function of age for pulsars in the ATNF catalog; Fermi-LAT detected pulsars are marked with black circles. Pulsar wind nebulae candidates are marked with red stars; LAT pulsars showing a significant off-pulse emission with a plausible magnetospheric origin are marked with blue squares. For pulsars with a distance range in Table 1, we use the geometric mean of the minimum and maximum values. Note that inaccurate distance estimates can introduce artificially low spin-down fluxes, which might account for the handful of pulsar detections below $10^{33} \mathrm{erg} \mathrm{s}^{-1} \mathrm{kpc}^{-2}$.

(A color version of this figure is available in the online journal.)

have a low $\gamma$-ray efficiency in the Fermi-LAT energy range, $\frac{L_{\text {PWN }}}{\dot{E}}<10^{-2}$, Vela-X, Crab and MSH 15-52 having even lower $\frac{L_{\mathrm{PWN}}}{\dot{E}}$ (see Figure 7 ). This implies that most of the 44 upper limits derived using 16 months of LAT observations are not yet constraining. However, for three objects $(\mathrm{J} 0659+1414$, J1833-1034, and J0205+6449) the upper limits in Figure 7 are well below $2 \times 10^{-3} \dot{E}$ suggesting that some pulsars are less efficient at producing GeV PWN flux than J1023-5746 and B1509-58. Table 2 indicates three potential PWNe candidates at $4 \sigma$ level: J0007+7303, J1028-5819, and J1709-4429. More data are needed to confirm the detection of these three sources and significantly probe the variation of the PWN flux with the spin-down power or with the ambient medium. One can also note two pulsars with significant emission in their off-pulse window and very high efficiency: J1836+5925 and J2021+4026. These two pulsars show a similar gamma-ray luminosity in the off-pulse and in the on-pulse intervals (renormalized to the total phase interval); their luminosities are greater than the spin-down power, assuming a uniform phase-averaged beaming across the sky. However, the association distances for the gamma-ray selected pulsars must be treated with caution.

Reporting the Fermi detection of HESS J1640-465, Slane et al. (2010) invoked an excess of low energy electrons to account for the $\mathrm{GeV}$ data and to explain the continuity in photon spectral index between the Fermi and HESS energy bands. While many of the upper limits on PWNe flux cataloged here are insufficiently constraining to rule out such a situation, we also see no evidence in support of such an electron spectrum for any object in the catalog. We also see no indication of dual electron populations, as proposed to explain Vela-X (Abdo et al. 2010c). The GeV and TeV peaks in Vela-X are comparable in flux, and this would put the GeV flux very near the Fermi sensitivity for

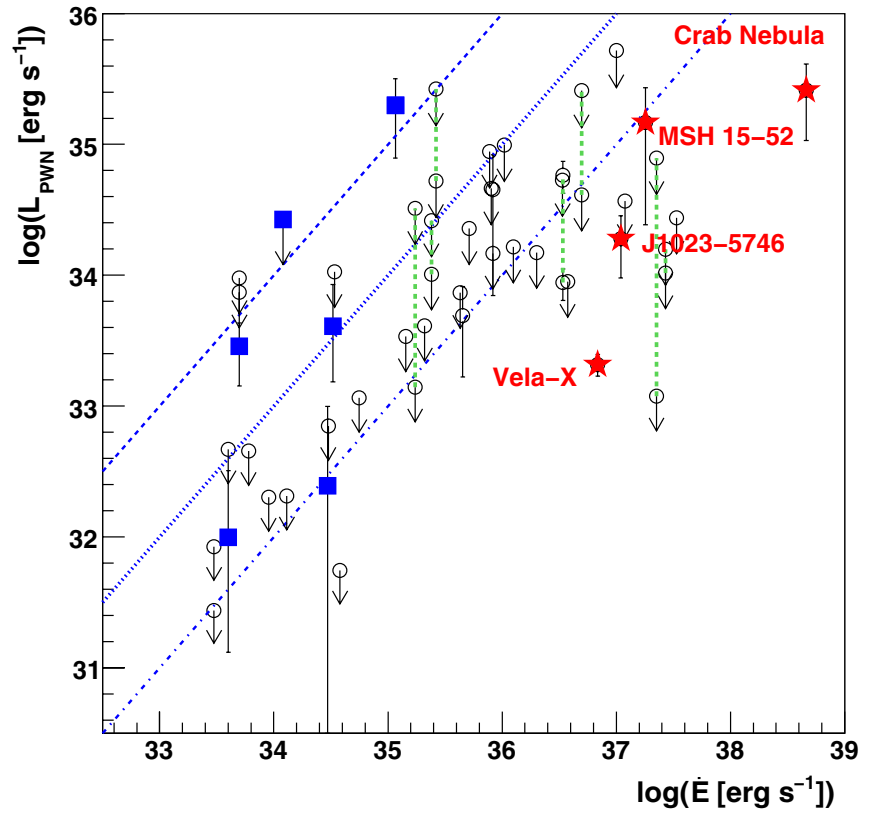

Figure 7. Dependence of the PWN luminosity and the pulsar spin-down power for LAT detected pulsars. Pulsar wind nebulae candidates are marked with red stars; pulsars showing a significant off-pulse emission with a plausible magnetospheric origin are marked with blue squares. Deleted and modified in the figure: potential candidates with test statistic values between 16 and 25 (discussed in Section 6.3) are marked with blue triangles. Only pulsars with estimated distances reported in Table 1 are plotted. Error bars take into account both the statistical uncertainties on the luminosity and the uncertainty on the distance of the pulsar. Lines correspond to constant $\gamma$-ray efficiency: $100 \%$ (dashed), $10 \%$ (dotted), and 1\% (dot-dashed). Pulsars with two distance estimates have two markers connected with green dashed error bars. The luminosity of the PWN in MSH 15-52 is taken from Abdo et al. (2010h).

(A color version of this figure is available in the online journal.)

a number of Vela-like pulsars with TeV counterparts: PSRs J1418-6058, J1420-6048, J1709-4429, and J1907+0602. Indeed, if most PWNe boast simple electron populations with a power-law index of $\sim 2.3$, the majority of these PWNe may prove elusive to Fermi.

The Fermi-LAT Collaboration acknowledges generous ongoing support from a number of agencies and institutes that have supported both the development and the operation of the LAT as well as scientific data analysis. These include the National Aeronautics and Space Administration and the Department of Energy in the United States, the Commissariat à l'Energie Atomique and the Centre National de la Recherche Scientifique/Institut National de Physique Nucléaire et de Physique des Particules in France, the Agenzia Spaziale Italiana and the Istituto Nazionale di Fisica Nucleare in Italy, the Ministry of Education, Culture, Sports, Science and Technology (MEXT), High Energy Accelerator Research Organization (KEK), and Japan Aerospace Exploration Agency (JAXA) in Japan, and the K. A. Wallenberg Foundation, the Swedish Research Council, and the Swedish National Space Board in Sweden.

Additional support for science analysis during the operations phase from the Istituto Nazionale di Astrofisica in Italy and the Centre National d'Études Spatiales in France is gratefully acknowledged.

The Parkes radio telescope is part of the Australia Telescope which is funded by the Commonwealth Government for operation as a National Facility managed by CSIRO. We thank our colleagues for their assistance with the radio timing observa- 
tions. The Green Bank Telescope is operated by the National Radio Astronomy Observatory, a facility of the National Science Foundation operated under cooperative agreement by Associated Universities, Inc. The Nançay Radio Observatory is operated by the Paris Observatory, associated with the French Centre National de la Recherche Scientifique (CNRS). The Lovell Telescope is owned and operated by the University of Manchester as part of the Jodrell Bank Centre for Astrophysics with support from the Science and Technology Facilities Council of the United Kingdom.

\section{REFERENCES}

Abdo, A. A., et al. (MILAGRO Collaboration) 2007, ApJ, 664, L91

Abdo, A. A., et al. 2009a, ApJ, 696, 1084

Abdo, A. A., et al. 2009b, ApJ, 706, L1

Abdo, A. A., et al. 2009c, ApJ, 706, 1331

Abdo, A. A., et al. 2009d, Science, 325, 840

Abdo, A. A., et al. (MILAGRO Collaboration) 2009e, ApJ, 700, L127

Abdo, A. A., et al. 2010a, ApJS, 187, 460

Abdo, A. A., et al. 2010b, ApJ, 713, 154

Abdo, A. A., et al. 2010c, ApJ, 713, 146

Abdo, A. A., et al. 2010d, ApJ, 708, 1254

Abdo, A. A., et al. 2010e, ApJ, 712, 957

Abdo, A. A., et al. 2010f, ApJ, 712, 1209

Abdo, A. A., et al. 2010g, ApJ, 711, 64

Abdo, A. A., et al. 2010h, ApJ, 714, 927

Abdo, A. A., et al. 2010i, ApJS, 188, 405

Abdo, A. A., et al. 2010j, ApJ, 720, 272

Abdo, A. A., et al. 2010k, ApJ, 722, 1303

Acciari, V. A., et al. 2009, ApJ, 703, L6

Aharonian, F., et al. 2002, A\&A, 393, L37

Aharonian, F., et al. (HESS Collaboration) 2006, A\&A, 456, 245

Aharonian, F., et al. (HESS Collaboration) 2007, A\&A, 467, 1075

Aharonian, F., et al. (HESS Collaboration) 2009, A\&A, 499, 723

Albert, J., et al. 2008, ApJ, 675, L25

Aliu, E. 2008, in AIP Conf. Proc. 1085, Proc. 4th Int. Meeting on High Energy

Gamma-Ray Astronomy, ed. F. A. Aharonian, W. Hofmann, \& F. Rieger (Melville, NY: AIP), 324

Anderhub, H., et al. 2010, ApJ, 710, 828

Atwood, W. B., et al. 2009, ApJ, 697, 1071

Bai, X.-N., \& Spitkovsky, A. 2010, ApJ, 715, 1282

Bednarek, W., \& Bartosik, M. 2005, J. Phys. G: Nucl. Part. Phys., 31, 1465

Bertsch, D. L., et al. 1992, Nature, 357, 306

Bietenholz, M. F., \& Bartel, N. 2008, MNRAS, 386, 1411

Blondin, J. M. 2001, ApJ, 563, 806

Blumenthal, G. R. 1970, Phys. Rev. D, 1, 1596

Bocchino, F., et al. 2005, A\&A, 442, 539

Camilo, F., et al. 2006, ApJ, 637, 456

Camilo, F., et al. 2009, ApJ, 705, 1

Cheng, K. S., Ruderman, M. A., \& Zhang, L. 2000, ApJ, 537, 964

Chevalier, R. A. 2005, ApJ, 619, 839

Cordes, J. M., \& Lazio, T. J. W. 2002, arXiv:astro-ph/0207156

D'Amico, N., et al. 2001, ApJ, 552, L45

Dame, T. M. 2007, ApJ, 665, L163

Daugherty, J. K., \& Harding, A. K. 1996, ApJ, 458, 278

de Jager, O. C., Ferreira, S. E. S., \& Djannati-Atai, A. 2008, in AIP Conf. Proc. 1085, Proc. 4th Int. Meeting on High Energy Gamma-Ray Astronomy, ed. F. A. Aharonian, W. Hofmann, \& F. Rieger (Melville, NY: AIP), 199

de Jager, O. C., \& Harding, A. K. 1995, in 24th Int. Cosmic Ray Conf., (Rome), 2, 528

De Rosa, A., Ubertini, P., Campana, R., Bazzano, A., Dean, A. J., \& Bassani, L. 2009, MNRAS, 393, 527

Djannati-Atai, A., et al. 2007, in Proc. 30th ICRC (Merida), 2, 823

Dyks, J., \& Rudak, B. 2003, ApJ, 598, 1201

Fichtel, C. E., Hartman, R. C., Kniffen, D. A., Thompson, D. J., Ogelman, H., Ozel, M. E., Tumer, T., \& Bignami, G. F. 1975, ApJ, 198, 163
Fujita, Y., Hayashida, K., Takahashi, H., \& Takahara, F. 2009, PASJ, 61, 1229

Fukui, Y., et al. 2009, PASJ, 61, L23

Gaensler, B. M., \& Slane, P. O. 2006, ARA\&A, 44, 17

Gelfand, J. D., Slane, P. O., \& Zhang, W. 2009, ApJ, 703, 2051

Green, D. A. 1986, MNRAS, 218, 533

Halpern, J. P., Camilo, F., Gotthelf, K. M., \& Helfand, D. J. 2001a, ApJ, 552, L125

Halpern, J. P., Gotthelf, E. V., Leighly, K. M., \& Helfand, D. J. 2001b, ApJ, 547, 323

Halpern, J. P., \& Holt, S. S. 1992, Nature, 357, 222

Hartman, R. C., et al. 1999, ApJS, 123, 79H

Helene, O. 1983, Nucl. Instrum. Methods.Phys. Res., 212, 319

Hobbs, G. B., Lyne, A. G., Kramer, M., Martin, C. E., \& Jordan, C. 2004, MNRAS, 353, 1311

Horns, D., Hoffmann, A. I. D., Santangelo, A., Aharonian, F. A., \& Rowell, G. P. 2007, A\&A, 469, L17

Kaaret, P., \& Cottam, J. 1996, ApJ, 462, L35

Kaplan, D. L., et al. 2005, PASP, 117, 643

Kelner, S. R., Aharonian, F. A., \& Bugayov, V. V. 2006, Phys. Rev. D, 74 034018

Kennel, C. F., \& Coroniti, F. V. 1984, ApJ, 283, 710

Kniffen, D. A., Fichtel, C. E., Hartman, R. C., Thompson, D. J., Ozel, M. E., Tumer, T., Bignami, G. F., \& Ogelman, H. 1975, in 14th ICRC (Munich), 1, 100

Kothes, R., Reich, W., \& Uyaniker, B. 2006, ApJ, 638, 225

Manchester, R. N., \& Taylor, J. H. 1977, Pulsars (San Francisco, CA: Freeman), 281

Mattox, J. R., et al. 1996, ApJ, 461, 396

Morsi, H. W., \& Reich, W. 1987, A\&AS, 69, 533

Muslimov, A. G., \& Harding, A. K. 2004, ApJ, 617, 471

Ng, C.-Y., \& Romani, R. W. 2008, ApJ, 673, 411

Pacini, F., \& Salvati, M. 1973, ApJ, 186, 249

Pavlov, G. G., Bhattacharyya, S., \& Zavlin, V. E. 2010, ApJ, 715, 66

Pellizzoni, A., et al. 2009, Science, 327, 663

Porter, T. A., Moskalenko, I. V., \& Strong, A. W. 2006, ApJ, 648, L29

Press, W. H., Teukolsky, S. A., Vetterling, W. T., \& Flannery, B. P. 1992, Numerical Recipes in C: The Art of Scientific Computing (2nd ed; Cambridge: Cambridge Univ. Press)

Ray, P. S., et al. 2010, ApJ, submitted (arXiv:1011.2468)

Roberts, M. S. E., Romani, R. W., Johnston, S., \& Green, A. 1999, ApJ, 515, 712

Romani, R. W. 1996, ApJ, 470, 469

Romani, R. W., \& Yadigaroglu, I. A. 1995, ApJ, 438, 314

Romani, R. W., \& Watters, K. 2010, ApJ, 714, 810

Romero, G. E., Benaglia, P., \& Torres, D. F. 1999, A\&A, 348, 868

Safi-Harb, S., Harrus, I. M., Petre, R., Pavlov, G. G., Koptsevich, A. B., \& Sanwal, D. 2001, ApJ, 561, 308

Salter, C. J., Reynolds, S. P., Hogg, D. E., Payne, J. M., \& Rhodes, P. J. 1989, ApJ, 338,171

Saz Parkinson, P. M., et al. 2010, ApJ, 725, 571

Slane, P., Castro, D., Funk, S., Uchiyama, Y., Lemiere, A., Gelfand, J. D., \& Lemoine-Goumard, M. 2010, ApJ, 720, 266

Slane, P., Helfand, D. J., Reynolds, S. P., Gaensler, B. M., Lemiere, A., \& Wang, Z. 2008, ApJ, 676, L33

Slane, P., Helfand, D. J., van der Swaluw, E., \& Murray, S. S. 2004, ApJ, 616, 403

Stephenson, F. R., \& Green, D. A. 2002, Historical Supernovae and their Remnants (New York: Oxford Univ. Press)

Theureau, G., et al. 2005, A\&A, 430, 373

Theureau, G., et al. 2010, A\&A, in press (arXiv:1010.4230)

Tian, W. W., \& Leahy, D. A. 2008, MNRAS, 391, L54

Van Etten, A., \& Romani, R. 2010, ApJ, 711, 1168

Venter, C., Harding, A. K., \& Guillemot, L. 2009, ApJ, 707, 800

Ward, J. E. 2008, in AIP Conf. Proc. 1085, High Energy Gamma-Ray Astronomy, ed. F. A. Aharonian, W. Hofmann, \& F. Rieger (Melville, NY: AIP), 301

Weiler, K. W., \& Panagia, N. 1978, A\&A, 70, 419

Weltevrede, P., et al. 2009, PASA, 27, 64

Whiteoak, J. B. Z., \& Uchida, K. I. 1997, A\&A, 317, 563

Yadigaroglu, I.-A., \& Romani, R. W. 1997, ApJ, 476, 347 